\title{
Efferent Association Pathways from the Rostral Prefrontal Cortex in the Macaque Monkey
}

\author{
Michael Petrides ${ }^{1,2}$ and Deepak N. Pandya ${ }^{3,4,5}$ \\ ${ }^{1}$ Montreal Neurological Institute, McGill University, Montreal, Quebec, Canada H3A 2B4, 2Department of Psychology, McGill University, Montreal, Quebec, \\ Canada H3A 1B1, ${ }^{3}$ Departments of Anatomy and Neurology, Boston University School of Medicine, Boston, Massachusetts 02118, ${ }^{4}$ Department of \\ Neurology, Beth Israel Deaconess Medical Center, Boston, Massachusetts 02215, and ${ }^{5}$ Edith Nourse Rogers Memorial Veterans Hospital, Bedford, \\ Massachusetts 01730
}

The different prefrontal cortical regions exert executive control over processing occurring in posterior cortical regions. We examined with the autoradiographic method, in the macaque monkey, the course and terminations of the efferent corticocortical connections of the rostral prefrontal region, the function of which is least understood. Three efferent streams of fibers organized into three distinct fasciculi convey rostral prefrontal influences on posterior cortical areas. These connections provide powerful insights into the cortical regions on which executive control is being exercised. The lateral stream of fibers via the extreme capsule targets the midsection of the auditory superior temporal region and the multisensory areas of the superior temporal sulcus, thus permitting control over the most integrated aspects of cognitive processing. The fibers coursing through the extreme capsule originating in areas 10 and 9 continue as part of the white matter of the superior temporal gyrus (i.e., the middle longitudinal fasciculus) to target the midportion of the superior temporal gyrus (areas TAa, TS2, and TS3) and adjacent multisensory area TPO within the upper bank of the superior temporal sulcus. Some of the fibers from areas 10 and 9 that enter the extreme capsule terminate in the ventral part of the insula. The dorsomedial limbic stream via the cingulate fasciculus targets the anterior and posterior cingulate cortex, as well as the retrosplenial cortex, allowing control over motivational and memory processes. A ventral limbic stream via the uncinate fasciculus targets the temporal proisocortex and the amygdala, indicating an additional powerful influence over the emotional motivational sphere.

Key words: monkey; frontal cortex; prefrontal cortex; association pathways; autoradiography; area 10; connections

\section{Introduction}

The role of the rostral part of the primate prefrontal cortex in cognitive processing is one of the least understood, and it has become the focus of considerable debate in recent years (Ramnani and Owen, 2004; Gilbert et al., 2006). The rostral prefrontal region includes frontopolar area 10 and the adjoining area 9, which occupies the rostral part of the superior frontal region. It is only during the last 5 years that a few suggestions about the functional contribution of the rostral prefrontal region have begun to emerge, mostly from functional neuroimaging work on human subjects. It has been suggested that it may be involved in some of the most complex control processes, such as branching from one task to another and integrating the outcomes of several cognitive operations (Ramnani and Owen, 2004; Gilbert et al., 2006). Unfortunately, not only is knowledge of the functional contribution of the rostral prefrontal cortex limited, but also relatively little is known of its efferent corticocortical connectivity, which could

Received May 27, 2007; revised Aug. 6, 2007; accepted Aug. 29, 2007.

This work was supported by Canadian Institutes of Health Research Grant MOP-14620 and Natural Sciences and Engineering Research Council of Canada Grant RGPIN 7466. We thank Helen Papageorgiou, Juergen Germann, and Veronika Zlatkina for preparing the illustrations.

Correspondence should be addressed to Michael Petrides, Montreal Neurological Institute, 3801 University Street, Montreal, Quebec, Canada H3A 2B4. E-mail: petrides@ego.psych.mcgill.ca.

DOI:10.1523/JNEUROSCI.2419-07.2007

Copyright $\odot 2007$ Society for Neuroscience $\quad$ 0270-6474/07/2711573-14\$15.00/0 provide major clues about its role in cognition by revealing the areas on which it primarily exerts its executive control.

The prefrontal cortex receives input from sensory-specific and multimodal post-Rolandic association cortical areas, as well as from limbic cortical regions (Barbas, 1988; Cavada and Goldman-Rakic, 1989; Carmichael and Price, 1995, 1996). The rostral part of the prefrontal cortex receives input via the cingulate and uncinate fasciculi, as well as the extreme capsule (Mufson and Pandya, 1984; Ungerleider et al., 1989; Carmichael and Price, 1995; Morris et al., 1999a), whereas the caudolateral prefrontal cortex receives most of its cortical input via the superior longitudinal, arcuate, and occipitofrontal fasciculi (Petrides and Pandya, 1984, 1988; Cavada and Goldman-Rakic, 1989). There are a few studies of the efferent association fibers originating from the rostral part of the prefrontal cortical region and which course via the cingulate fasciculus to terminate in the cingulate and retrosplenial region (Nauta, 1964; Goldman-Rakic et al., 1984; Mufson and Pandya, 1984; Morris et al., 1999b), but the pathways used by efferent fibers from the rostral frontal region on their course to the lateral post-Rolandic association cortex have not been the subject of systematic investigation.

The present study examined the efferent association fibers originating from the rostral part of the prefrontal cortex of the macaque monkey with the autoradiographic technique. This method, which involves the injection of radioactively labeled iso- 
topes in a region of interest, provides a demonstration of the precise course and terminations of efferent fibers that originate from the injected region. In addition to injections of isotopes in areas 10 and 9, which constitute the region of interest (see Fig. 1), we injected isotopes in area 32 that borders areas 10 and 9 on the medial surface of the rostral frontal lobe and area 11 that borders area 10 on the orbital surface of the frontal lobe so that the specificity of the connections of the rostral prefrontal cortex will be highlighted.

\section{Materials and Methods}

Surgery. Injections of radioactively labeled amino acids were placed in different parts of the rostral prefrontal cortex in seven rhesus monkeys (Macaca mulatta). The care and use of animals were in accordance with the guidelines of the National Institutes of Health. The animals were immobilized with ketamine hydrochloride $(10 \mathrm{mg} / \mathrm{kg}$ ) and then deeply anesthetized with sodium pentobarbital $(30 \mathrm{mg} / \mathrm{kg}$ ) (Sigma, St. Louis, MO) administered intravenously. A craniotomy was then performed, under aseptic surgical techniques, over the rostral part of the frontal lobe. In each case, an attempt was made to place two juxtaposed isotope injections into an architectonic area in the rostral prefrontal cortex according to the parcellation of Petrides and Pandya (1994). The intracortical injections consisted of radioactively labeled amino acids $\left({ }^{3} \mathrm{H}\right.$-leucine and/or proline; volume range, $0.4-1.0 \mu \mathrm{l}$; specific activity range, $40-80$ $\mu \mathrm{Cu}$, aqueous solution; New England Nuclear Brand Radiochemicals from PerkinElmer, Norwalk, CT). After survival periods ranging from 7 to $10 \mathrm{~d}$, the animals were deeply anesthetized with sodium pentobarbital and perfused transcardially with physiologic saline, followed by a $10 \%$ formalin solution.

The brains were divided into two blocks by a coronal cut and photographed from all angles. They were subsequently embedded in paraffin and sectioned at $16 \mu \mathrm{m}$ thickness. The brains were then processed for autoradiography according to the technique described by Cowan et al. (1972). The exposure times varied between 3 and 6 months. At monthly intervals, trial sections were developed for identification of optimal radiolabeling. The sections were also counterstained with thionine to permit identification of the architectonic areas. A series of coronal sections of the hemisphere were examined microscopically with dark-field illumination. The labeled fibers in the white matter and the terminal labeling in the cerebral cortex and subcortical structures were recorded with the aid of an X-Y plotter (Hewlett Packard, Corvallis, OR) that was electronically coupled to the stage of the microscope (Aristoplan; Leitz, Wetzlar, Germany). This information was then used to reconstruct the injection and termination sites, as well as the path of the labeled fibers. The cytoarchitectonic boundaries of the projection areas within the cerebral cortex, as well as the sites of origin of the pathways within the prefrontal cortex were established in the experimental material under light field illumination.

The distribution of the terminations of the labeled fibers was transferred onto two-dimensional (2D) reconstructions (i.e., flattened views) of the lateral, medial, and ventral surfaces of the examined cerebral hemispheres. These two-dimensional reconstructions of the hemispheres were made using a precision technical drawing software program (AutoSketch, Release 7; Autodesk). To minimize the distortion in the normal view of the cerebral hemisphere, which inevitably follows such flattened maps, we unfolded the cortex lying within the major sulci separately. These unfolded sulci are presented next to the lateral and medial views of the hemispheres so that the reader can appreciate the details of the terminations within the sulci. On the coronal sections of the hemisphere that was to be reconstructed, we traced the distance from the midline (i.e., the border of the lateral with the medial surface of the hemisphere) to the first sulcus encountered laterally. We then measured the distance from that sulcus to the next sulcus and so on until the lateral-to-ventral edge of the hemisphere was reached. These measurements were used for the lateral surface reconstruction. For the reconstruction of the medial surface of the frontal lobe, we measured the distance from the dorsal-most part of the midline to the first sulcus encountered ventrally and then to the next sulcus until the ventral-most

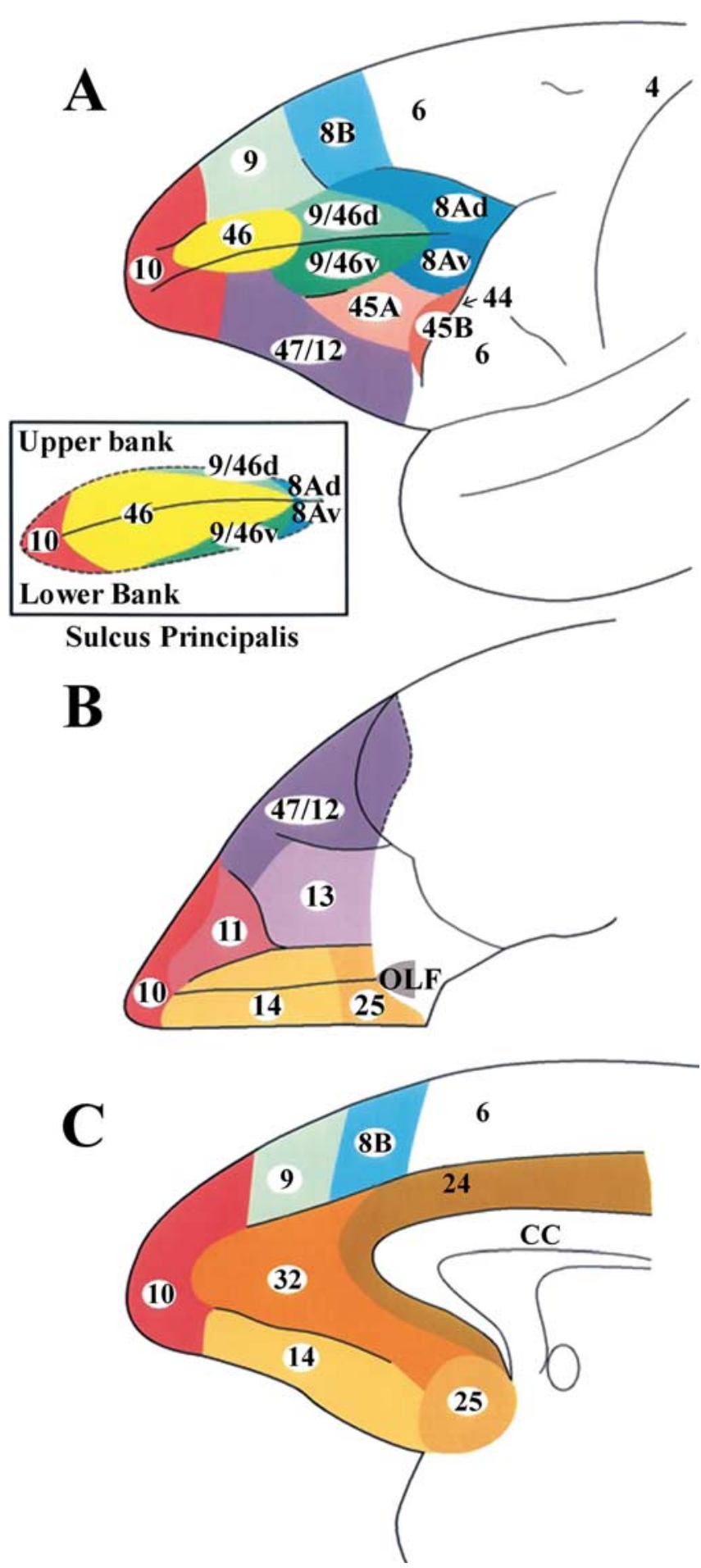

Figure 1. Cytoarchitectonic map of the lateral $(\boldsymbol{A})$, orbital $(\boldsymbol{B})$, and medial $(\boldsymbol{C})$ surfaces of the frontal lobe of the monkey according to the study by Petrides and Pandya (1994). $A$, Inset, The cortex in the banks of the sulcus principalis.

part of the medial surface was reached. The orbital surface of the frontal lobe was reconstructed by measuring the distance, on each coronal section, from the midline to the first sulcus encountered laterally (i.e., the medial orbital sulcus) and from there to the lateral orbital sulcus and then to the ventral-to-lateral edge of the hemisphere. The medial/ventral surface of the temporal lobe was included with the reconstruction of the medial surface of the hemisphere and the origin of the measurements was the hippocampal sulcus. The measurements obtained for the lateral, medial, and orbital surfaces, as well as the cortex within the sulci, were thus 


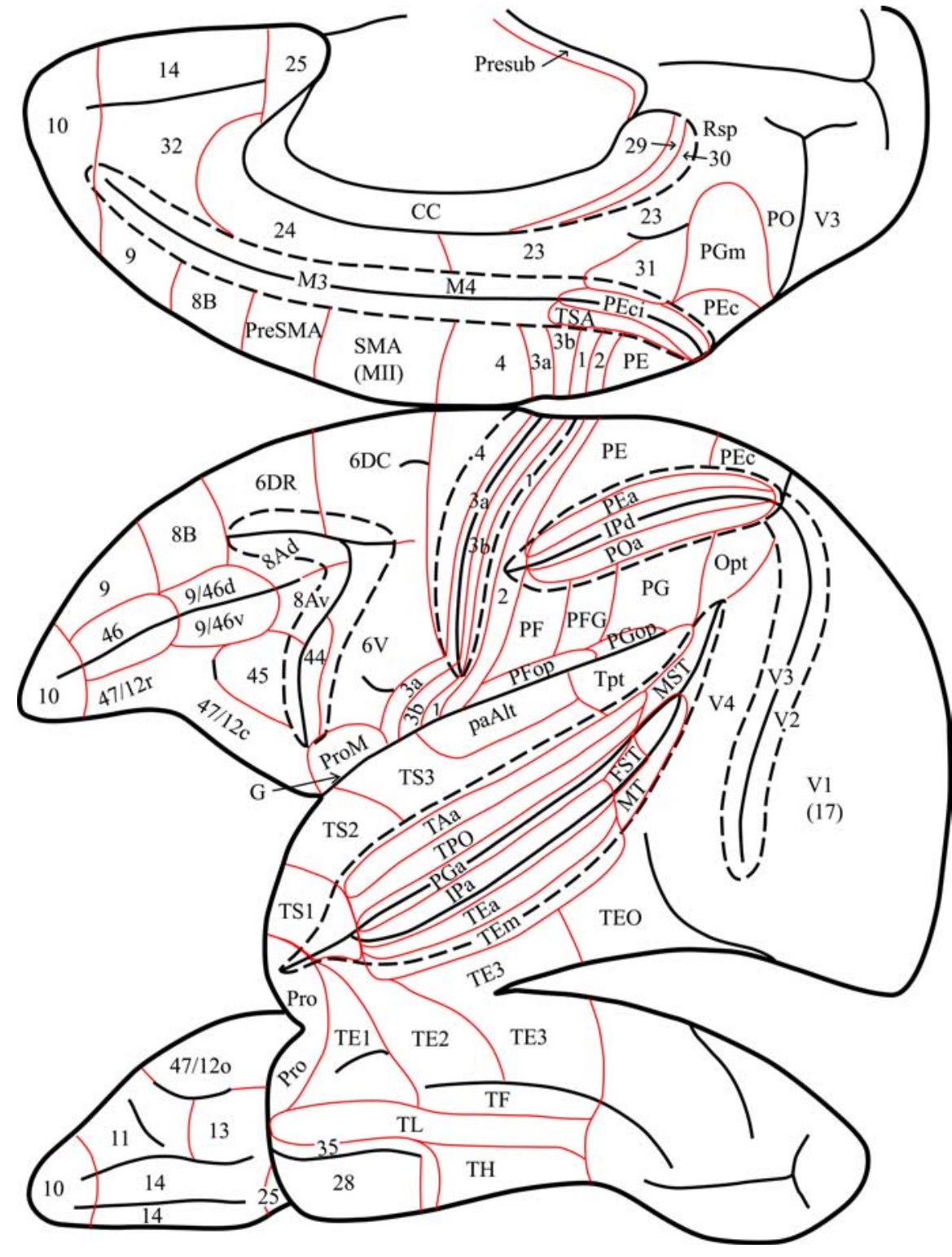

Figure 2. Diagram of the medial, lateral, and ventral views of the monkey left cerebral hemisphere to show the location of the architectonic areas used in the present article to describe the results. Interrupted lines indicate the lips of the opened sulci to show architectonic areas within them. Red lines show the borders of architectonic areas. Note the interrupted line that indicates removal of the posterior part of the corpus callosum to show the retrosplenial areas 29 and 30 that lie within the callosal sulcus.

the investigator's choice. All views are automatically updated as the cursor is moved to any point in a given section and the investigator can mark any point in the MRI volume. The markings of the charted fibers on a series of contiguous two-dimensional sections could then be transferred onto comparable coronal sections of the standard MRI brain using local details in the anatomy of the sections. Once the marked fibers from the charted case were transferred into the MRI brain, they could be reconstructed and viewed on the three-dimensional rendering of the standard MRI brain, permitting a clear visualization of the course of the entire pathway from various angles. This $3 \mathrm{D}$ reconstruction provides a clearer appreciation of the course of the various pathways within the white matter of the cerebral hemisphere and is illustrated in Figures 15 and 16. The 3D hemispheric view provides an impression of the trajectories of the identified association pathways, whereas the coronal sections indicate the location of these pathways at different levels.

Terminology, architectonic parcellation, and borders. The architectonic delineation of the prefrontal cortex was determined according to the criteria of Petrides and Pandya (1994) (Fig. 1 ), the posterior parietal cortex according to Pandya and Seltzer (1982), the superior temporal gyrus according to Pandya and Sanides (1973), the inferotemporal cortex and the cortex of the superior temporal sulcus according to Seltzer and Pandya (1978), and the posterior parahippocampal gyrus according to Rosene and Pandya (1983). The parcellation of the cingulate cortex, and that of the retrosplenial cortex, which lies on the dorsal aspect of the corpus callosum, follows the study by Vogt et al. (1987). The location of these and other cortical architectonic areas are shown in Figure 2. The atlas of the macaque monkey brain by Paxinos et al. (2000) also displays the limits of the architectonic areas used in the present analysis.

It should be noted that, in the present article, the association fibers that course in the white matter of the cingulate gyrus are referred to as the cingulate fasciculus. The term cingulum or cingulum bundle has sometimes been used to refer to all of the white matter underneath the cingulate gyrus. This term, however, is confusing because the cingulate white matter contains

a series of line segments (the y coordinates) arranged in the anteroposterior direction ( $x$ coordinates). In separate spreadsheets, the points $(x, y$ coordinates) were plotted and joined together to reconstruct the twodimensional flattened outlines of surfaces of the hemisphere and the sulci.

The labeled long association fibers that were traced on coronal sections of selected cases were transferred onto the corresponding coronal sections of a monkey brain (male, M. mulatta) that was scanned with magnetic resonance imaging (MRI). A high-resolution T1-weighted 3D MRI anatomical image of this monkey brain was acquired from a $1.5 \mathrm{~T}$ Siemens Sonata Vision scanner (repetition time, $22 \mathrm{~ms}$; echo time, $9.2 \mathrm{~ms}$; flip angle, $30^{\circ} ; 1.0 \times 1.0 \times 1.0 \mathrm{~mm}$; coronal slices, $1.0 \mathrm{~mm}$ in thickness). Three-dimensional surface reconstructions of this brain were obtained by using an automated, model-based, surface deformation algorithm (MacDonald et al., 1994). The position of the labeled fibers was marked on the MRI monkey brain using DISPLAY, an interactive threedimensional imaging software package (MacDonald, 1996) that allows the MRI file to be viewed and marked simultaneously in the coronal, horizontal, and sagittal planes, as well as in one other arbitrary plane of cortical association fibers, as well as subcortical and commissural fibers. To be more specific, Mufson and Pandya (1984) restricted the term cingulum bundle to the subcortical fibers that originate from and are directed to the cingulate cortex (the thalamocingulate and cingulothalamic fibers), while referring to the cortical fibers as the cingulate fasciculus. The subcortical fibers, in general, course in the central and ventral part of the cingulate white matter, whereas the long association fibers (cingulate fasciculus) occupy a more dorsal and medial position around the subcortical fibers.

\section{Results}

\section{Case 1 (area 10)}

In this case, the isotope injection was placed in the frontopolar cortex and involved the lateral, medial, and orbital area 10 (Fig. 3). A large number of labeled fibers were observed in the immediately adjacent white matter. Terminal label was noted in the adjacent cortical areas 9, 46, 32, 14, and 11. Further caudally 
within the lateral and medial frontal cortex, discrete clusters of terminal label were seen in areas $8 \mathrm{~B}, 8 \mathrm{Ad}, 6 \mathrm{DR}, 47 / 12,45,24$, and 25. Likewise, in the orbital frontal cortex, terminal label was seen in areas $47 / 12$, $11,13,14$, and the orbital proisocortex (Pro). In all these areas, the terminations occurred mainly in a columnar manner.

Approximately at the level of the midpoint of the sulcus principalis, the labeled fibers in the white matter began to separate into discrete clusters. These could be divided into the commissural fibers (CC), the long association fibers [i.e., the uncinate fasciculus (UF), the extreme capsule (Extm C), the cingulate fasciculus (CING F)], and the subcortical bundles (SB) (i.e., the striatal and thalamo-pontine bundles) (Fig. 4, section 2). The commissural fibers separated from the main bulk of the labeled fibers as they moved medially to enter the ventral part of the genu of the corpus callosum (Fig. 4, section 2). The fibers directed to the thalamus and the pons entered the anterior limb of the internal capsule. A major contingent of these fibers entered the rostral pole of the thalamus and terminated within the dorsomedial, intralaminar, and pulvinar nuclei. Another contingent of these fibers continued ventrally toward the cerebral peduncle directed to the pontine nuclei. Some of these fibers terminated in the hypothalamic region, but this projection was minor. The latter finding is consistent with Öngür et al. (1998) and Rempel-Clower and Barbas (1998), who reported prefrontal input to the hypothalamus primarily from the caudal orbitofrontal cortex.

At the level of the frontal horn of the lateral ventricle, the striatal fibers surrounded the head of the caudate nucleus and began to terminate within it. Further back at the beginning of the anterior limb of the internal capsule, the striatal fibers separated into the subcallosal fascicle [Muratoff bundle (MB)], which coursed dorsal to the caudate nucleus terminating as discrete patches within its head and body of the caudate nucleus, and the external capsule (EC) terminating within the rostral part of the putamen (Fig. 4, sections 3,4$)$.

The labeled long association fibers could be divided into a dorsal and a ventral group. The dorsal group entered the cingulate fasciculus and coursed caudally to terminate into area 24 , area 23 , and the retrosplenial cortex in area 30 (Figs. $3 B, 4$ ). The ventral group of long association fibers coursed in the white matter just above the orbital frontal cortex (Fig. 4, sections 2, 3). At the caudal-most part of the orbital frontal cortex, these fibers moved laterally. Many of them terminated in the ventral part of the claustrum, whereas others entered the uncinate fasciculus (ventral to the
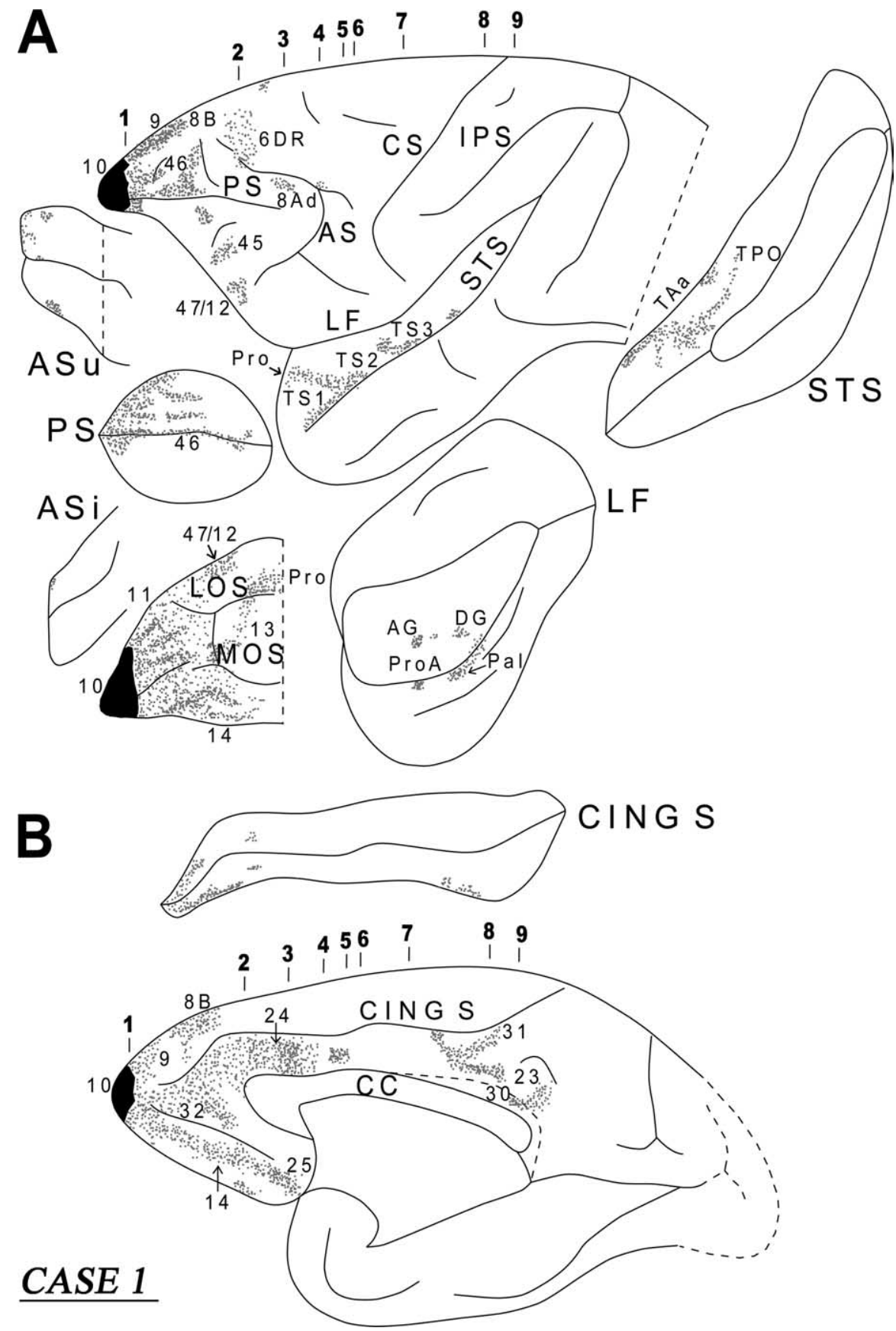

Figure 3. Diagrammatic representation of the unfolded lateral $(\boldsymbol{A})$ and medial $(\boldsymbol{B})$ surfaces of the cerebral hemisphere in case 1 with isotope injection in frontopolar area 10 (shown in solid black) and the resulting distribution of terminal label (shown as dots). In $\boldsymbol{A}$, the principal sulcus, the upper and inferior limbs of the arcuate sulcus, the lateral fissure, and the superior temporal sulcus have been opened up to show the terminal label in their banks. In $\boldsymbol{B}$, the cingulate sulcus has been opened up. AF, Arcuate fasciculus; AMG, amygdala; $A S$, arcuate sulcus; $A S i$, arcuate sulcus (inferior ramus); $A S u$, arcuate sulcus (upper ramus); CING $S$, cingulate sulcus; $C F$, calcarine fissure; $C S$, central sulcus; Extm F, extreme capsule fasciculus; FOF, fronto-occipital fasciculus; IC, internal capsule; LF, lateral fissure; IPS, intraparietal sulcus; IOS, inferior occipital sulcus; LOS, lateral orbital sulcus; MOS, medial orbital sulcus; OTS, occipitotemporal sulcus; Presub, presubiculum; PS, sulcus principalis; RS, rhinal sulcus; Rsp, retrosplenial cortex; SLF I, superior longitudinal bundle I; SLF II, superior longitudinal bundle II; SLF III, superior longitudinal bundle III; St B, striatal bundle; STS, superior temporal sulcus.

claustrum) and the ventral part of the extreme capsule (lateral to the claustrum) (Fig. 4, sections 4,5). Some of the labeled fibers running in the uncinate fasciculus coursed through the limen insulae to enter the white matter of the rostral-most part of the superior temporal gyrus and terminated in the temporal proisocortex and in area Ts1 in a columnar manner (Fig. 4, section 4). 


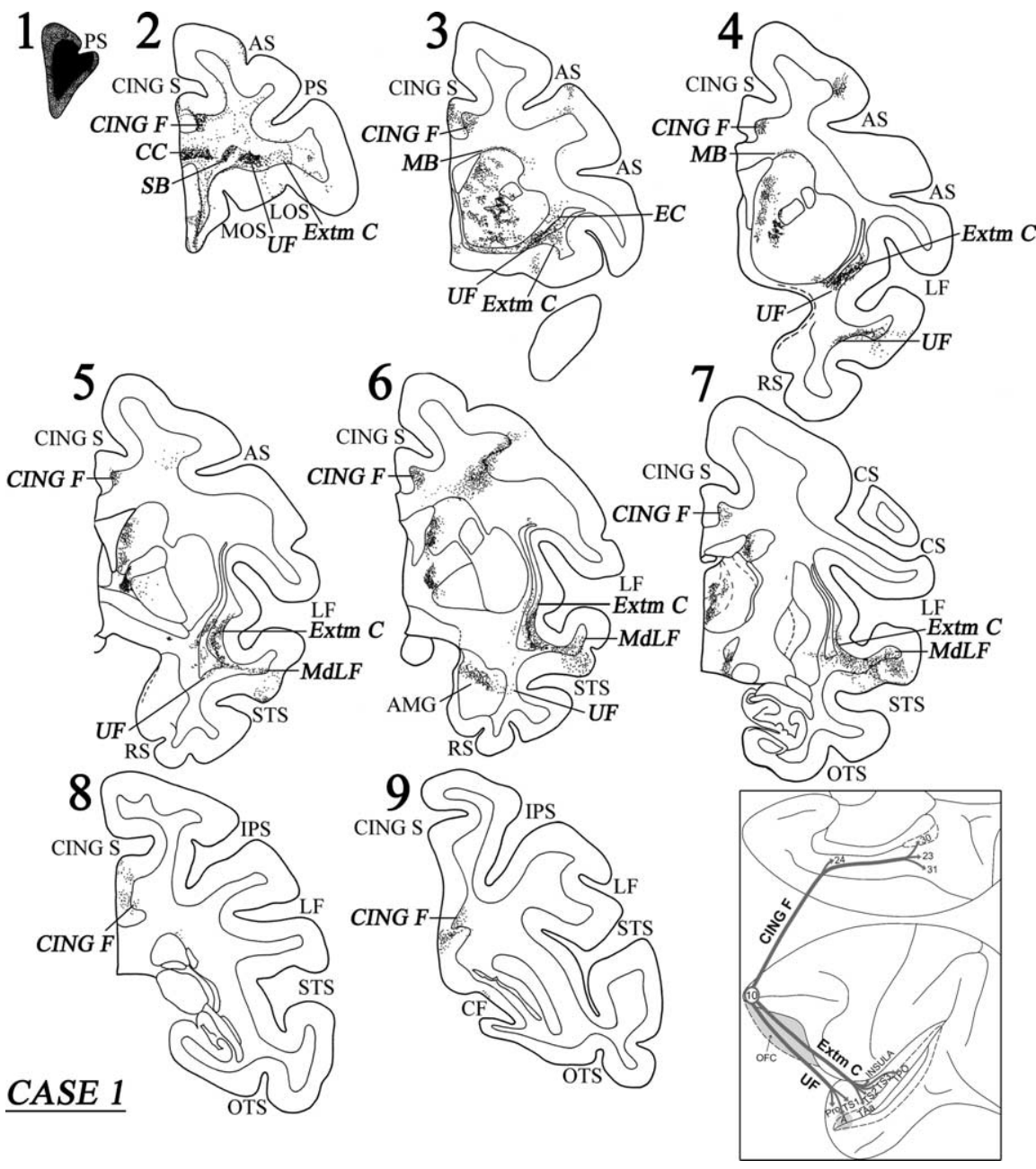

Figure 4. Coronal sections (1-9), at the levels indicated in Figure $3 A$, show the injection site and the labeled pathways in the white matter in case 1. The inset summarizes schematically the origin, trajectories, and terminations of the long association pathways observed in this case. For abbreviations, see Figure 3.

Another contingent of the labeled fibers in the uncinate bundle moved ventromedially to enter the amygdala (Fig. 4, section 6). These fibers terminated predominantly in the dorsal part of the basolateral nucleus (magnocellular part), in the dorsal part of the basomedial nucleus (magnocellular part), and in the cortical nucleus of the amygdala (for location of these nuclei, see Paxinos et al., 2000).

Labeled fibers coursing through the ventral part of the extreme capsule entered the white matter of the middle part of the superior temporal gyrus, merging with the fibers of the middle longitudinal fasciculus (MdLF), and terminated into areas TS2 and TS3 of the superior temporal gyrus and areas TAa and TPO in the superior temporal sulcus (Figs. 3A, 4, sections 5-7). The MdLF is a corticocortical bundle in the white matter of the superior temporal gyrus consisting of fibers connecting inferior parietal cortex with cortex primarily in the upper bank of the superior temporal sulcus and was first defined by Seltzer and Pandya (1978). The terminations in the superior temporal region of fibers running in the extreme capsule were mostly columnar in nature. In area TPO, there were increased terminations in layer I. Some of the labeled fibers in the extreme capsule terminated in the ventral part of the insula and in area $\mathrm{PaI}$ of the lateral fissure. The diagram in the inset of Figure 4 summarizes schematically the three long association pathways originating from area 10, namely the cingulate fasciculus (CING F), the UF, and the extreme capsule fiber system (Extm C).

Case 2 (ventral and orbital area 10)

In this case, the injection involved the ventral portion of area 10 mainly in the orbital cortex (Fig. 5). Immediately caudal to the injection, the white matter contained a significant number of labeled fibers, mostly in the ventral part of the white matter. Terminal label was seen in the adjacent cortical areas 9, 46, 10, 11, and 14. Further caudally in the frontal lobe, terminal label was predominantly observed in areas $47 /$ $12,14,13,32$, and 25. Some label was also noted dorsally in area 8Ad. These terminations were predominantly in a columnar manner. The course of the labeled callosal, long association, and projection fibers in this case were similar to those of case 1 , except for the lack of labeled fibers in the cingulate fasciculus in the present case, suggesting that the cingulate fasciculus fibers originate from the dorsal part of area 10 .

\section{Case 3 (lateral and medial area 9)}

In this case, the injection involved the lateral and medial parts of area 9 and invaded the caudal part of area 10 (Fig. 6). Short association fibers ran in the white matter of the frontal lobe to terminate, in the lateral surface, in areas $10,46,9,8 \mathrm{~B}$, and 47 / 12. On the medial surface, the fibers terminated in areas $10,14,32,24$, and 25 . On the orbital surface, the labeled fibers terminated in areas $47 / 12,11,14,13$, and the orbital proisocortex. All these terminations were columnar in nature. From the injection site, a thick cord of labeled fibers emerged in the dorsal prefrontal white matter (Fig. 7, section 2). These fibers then separated into a medial and a lateral segment. The medial segment entered the dorsal part of the genu of the corpus callosum. A component of the lateral segment surrounded the head of the caudate nucleus. These fibers, at the level of the anterior limb of the internal capsule, separated into a dorsal part entering the $\mathrm{MB}$ and terminated in the head and body of the caudate nucleus and a ventral part that entered the external capsule and terminated in the rostroventral part of the putamen and the ventral part of the claustrum (Fig. 7, sections 3,4). Another contingent of labeled fibers originating from the lateral component of the cord entered the internal capsule and terminated in the dorsomedial nucleus, the pulvinar, and the nucleus limitans of the thalamus. Some terminal label was also noted in the superior colliculus and the periacqueductal gray. Only minor label was noted in the hypothalamus consistent with observations by Öngür et al. (1998) and Rempel-Clower and Barbas (1998), who observed that hypothalamic input from the frontal cortex originates primarily from the caudal orbitofrontal region. Other fibers from this lateral component proceeded ventrally toward the pontine nuclei via the cerebral peduncle.

Two bundles of labeled long association fibers could be discerned in this case. There was a ventral bundle of labeled fibers 

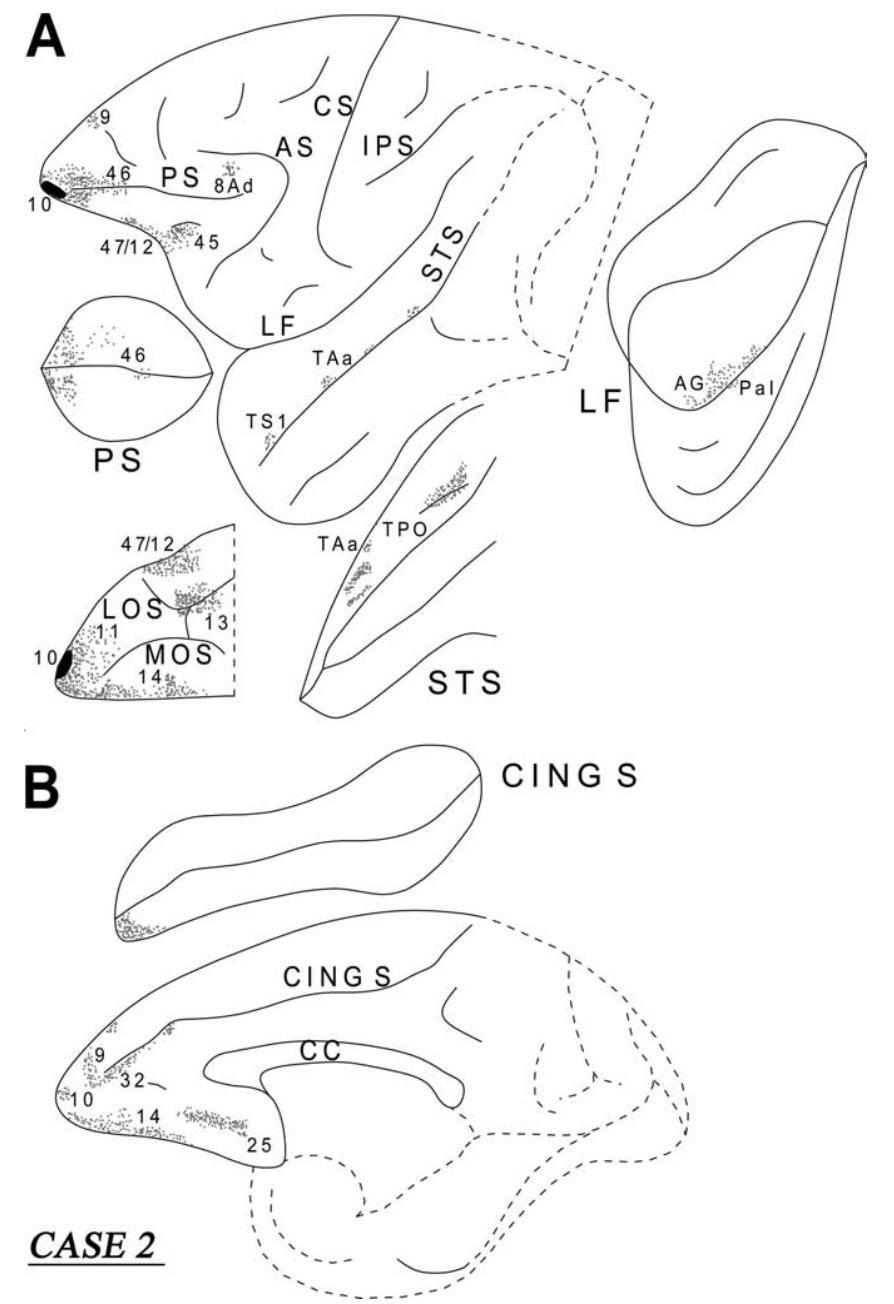

Figure 5. Diagrammatic representation of the unfolded lateral $(\boldsymbol{A})$ and medial $(\boldsymbol{B})$ surfaces of the cerebral hemisphere in case 2 with isotope injection in the ventral part of the frontopolar area 10 (shown in solid black) and the resulting distribution of terminal label (shown as dots). In $A$, the principal sulcus, the lateral fissure, and the superior temporal sulcus have been opened up to show the terminal label in their banks. In $\boldsymbol{B}$, the cingulate sulcus has been opened up. For abbreviations, see Figure 3.

that coursed through the lower part of the extreme capsule and entered the white matter of the superior temporal gyrus (Fig. 7, sections 4-6). From there, the fibers terminated in areas TAa and TPO in the upper bank of the superior temporal sulcus. The dorsal group of labeled fibers entered the cingulate fasciculus and terminated in areas 24,23 , and 31 , as well as in retrosplenial area 30 (Fig. 7, sections 4-9). Some labeled fibers were also noted in area TF of the parahippocampal gyrus (Fig. $6 B$ ).

\section{Case 4 (area 9)}

In this case, the isotope injection was placed in area 9 (Figs. 8, 9). Rostral to the injection site, terminal label was noted in the dorsal and ventral parts of area 10. At the level of the injection site, terminal label was noted in the dorsal and ventral banks of the sulcus principalis (areas 46 and 9/46). Caudal to the injection site, within the lateral frontal cortex, the terminal label was noted in areas $46,9 / 46 \mathrm{~d}, 8 \mathrm{~B}, 8 \mathrm{Ad}, 6 \mathrm{DR}$, and 47/12. On the medial surface of the frontal cortex, discrete columnar patches of terminations were noted in areas $32,24,9$, and $8 \mathrm{~B}$. On the orbital surface, terminations were observed in areas $47 / 12,11,10,14,13$, and the orbital proisocortex.
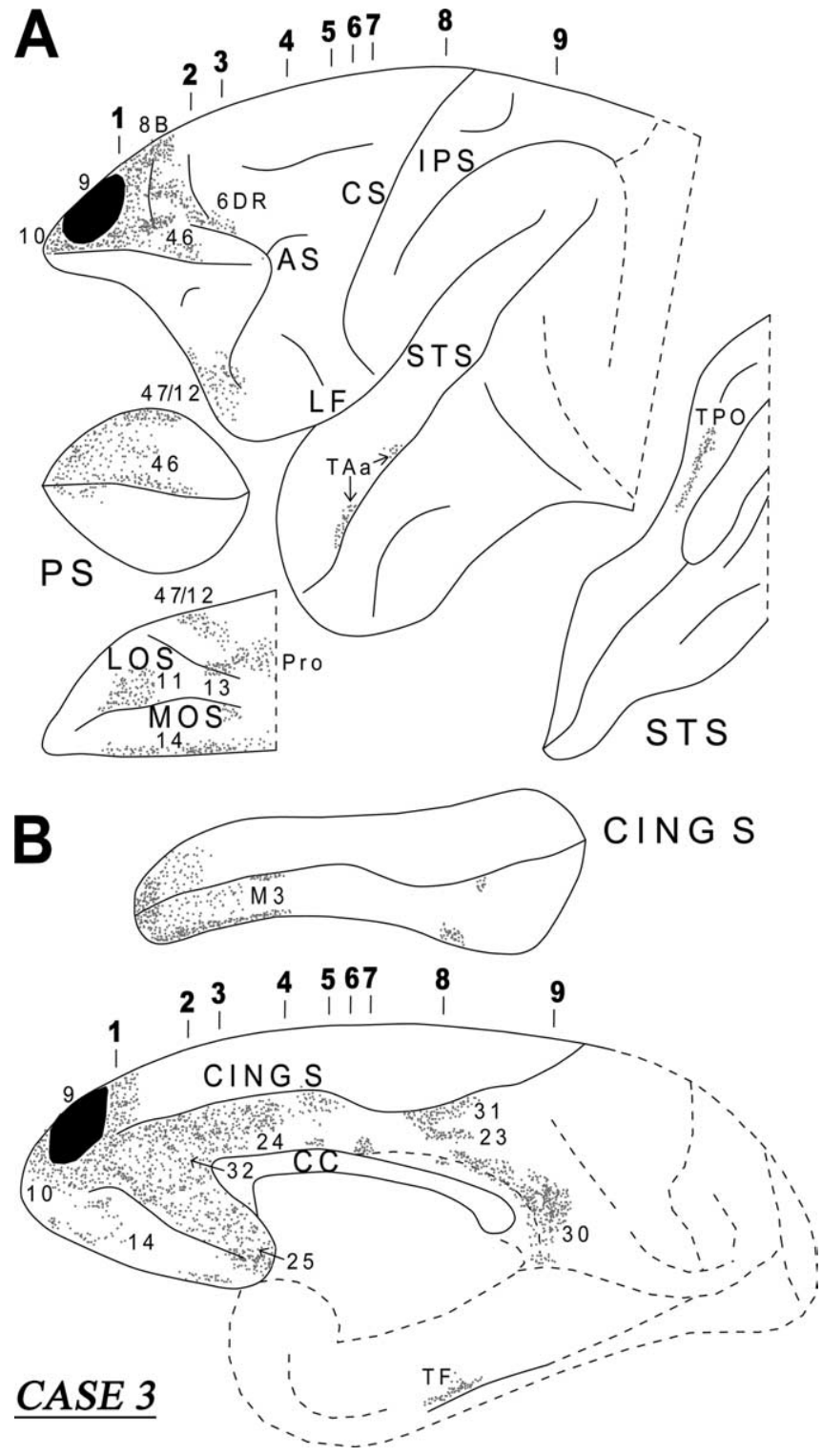

Figure 6. Diagrammatic representation of the unfolded lateral $(\boldsymbol{A})$ and medial $(\boldsymbol{B})$ surfaces of the cerebral hemisphere in case 3 with isotope injection in the lateral and medial parts of area 9 (shown in solid black) and the resulting distribution of terminal label (shown as dots). The principal sulcus and the superior temporal sulcus $(\boldsymbol{A})$ and the cingulate sulcus $(\boldsymbol{B})$ have been opened up to show the terminal label in their banks. For abbreviations, see Figure 3.

A dense cord of fibers, originating from the injection site, coursed ventrally in the white matter. At the level of the frontal horn of the lateral ventricle, these fibers separated into three groups. One group of fibers entered the dorsal part of the genu of the corpus callosum to terminate in the contralateral hemisphere. Another group of labeled fibers surrounded the head of the caudate nucleus at its rostral extremity and divided into two components at the level of the internal capsule, namely the bundle of Muratoff, dorsally, and the external capsule, ventrally (Fig. 9, section 4). The fibers of the bundle of Muratoff coursed caudally and terminated in distinct patches throughout the head and the body of the caudate nucleus. The labeled fibers in the external capsule terminated in the rostral part of the putamen, in the nucleus accumbens, and in the claustrum (Fig. 9, section 4).

The third group of fibers from the cord gathered lateral to the head of the caudate nucleus and entered the anterior limb of 

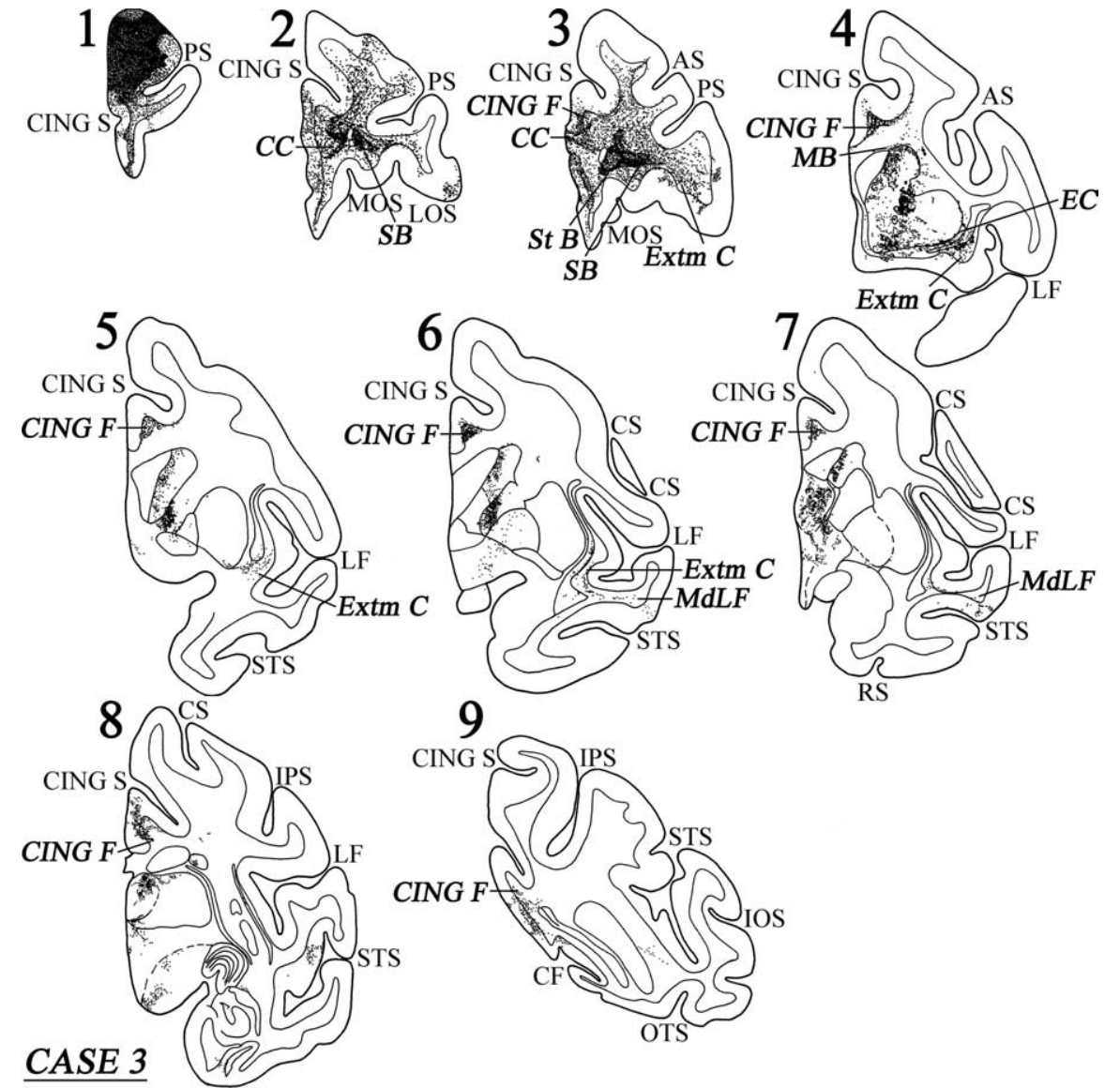

Figure 7. Coronal sections (1-9), at the levels indicated in Figure $6 A$, show the injection site and the labeled pathways in the white matter in case 3. For abbreviations, see Figure 3.

the internal capsule and coursed caudally as far as the genu of the internal capsule. Here, these fibers divided into two groups. One group of fibers moved medially into the thalamus, whereas the other group coursed ventrally to enter the cerebral peduncle and terminated in the pontine nuclei. There was also minor input to the hypothalamus. The thalamic fibers terminated principally in the dorsomedial thalamic nucleus, the intralaminar nuclei, and the pulvinar. Some of these fibers entered the rostral brainstem and terminated in the superior colliculus and the periaqueductal gray.

The long association fibers were organized into two principal groups. One group of fibers coursed through the cingulate fasciculus and the other through the extreme capsule. The fibers in the cingulate fasciculus coursed caudally and terminated in area 24, in the cingulate motor areas M3 and M4, and, caudally, in the posterior cingulate areas 23 and 31, as well as the retrosplenial area 30 (Fig. $8 B$ ). Some of these fibers terminated into the caudal end of the presubiculum (Fig. 9, section 7). The long association fibers that traveled via the extreme capsule entered the white matter of the superior temporal gyrus (MdLF) and terminated in area PaI in the lateral fissure and areas TAa and TPO in the superior temporal sulcus (Fig. 9, sections 6, 7). Some of the labeled fibers in the extreme capsule terminated in the dysgranular insula (Fig. 9, section 7). The diagram in the inset of Figure 9 summarizes schematically the two long association pathways originating from area 9, namely the cingulate fasciculus and the extreme capsule fiber system.

\section{Case 5 (medial area 9)}

The injection in this case involved the medial part of area 9 (Fig. $10 \mathrm{~B}$ ). On the medial surface of the frontal lobe, the labeled fibers terminated in areas $10,32,24,25$, and $8 \mathrm{~B}$. On the lateral surface, terminal label was noted in area $10,9,46,8 \mathrm{~B}, 6 \mathrm{DR}$, and $47 / 12$ (Fig. $10 A$ ). On the orbital surface, labeled terminations were noted in areas $47 / 12,11$, and 14 . From the injection site, a dense cord of labeled fibers coursed around the rostral part of the cingulate sulcus. These fibers then divided into two groups. The medial group entered the dorsal part of the genu of the corpus callosum to terminate in the opposite hemisphere. The lateral group divided into fibers directed to the striatum and fibers that entered the anterior limb of the internal capsule. The striatal fibers surrounded the anterior portion of the head of the caudate nucleus and, at the level of the anterior limb of the internal capsule, entered the Muratoff bundle to terminate into the head and body of the caudate nucleus. Other striatal fibers proceeded ventrolaterally in the external capsule and terminated in the putamen and the claustrum. Another contingent of labeled fibers entered the anterior limb of the internal capsule. These fibers were directed to the dorsomedial nucleus of the thalamus and the cerebral peduncle on their way to the pontine nuclei. The labeled long association fibers were restricted to the cingulate fasciculus. These fibers terminated in the posterior cingulate area 23 and the retrosplenial area 30 (Fig. $10 B)$.

\section{Case 6 (area 32)}

The injection in this case involved area 32 with some extension into the adjacent area 24 (Fig. $11 \mathrm{~B}$ ). On the medial surface of the frontal cortex, the labeled fibers terminated in areas $10,9,8 \mathrm{~B}, 24$, and 25 (Fig. $11 B$ ). On the lateral surface of the frontal cortex, terminal label was noted in areas 10, 46, 9, 8B, and 6DR (Fig. $11 A)$. In the orbitofrontal cortex, labeled terminations were observed in areas 11,14, 47/12,13, and the orbital proisocortex.

A dense cord of labeled fibers was observed in the white matter emerging from the injection site. Further caudally, this cord of fibers separated into a medial and a lateral group. The medially directed group of fibers entered the genu of the corpus callosum to terminate in the opposite hemisphere. The lateral group of fibers from the cord separated into a component that surrounded the head of the caudate nucleus. At the level of the anterior limb of the internal capsule, the corticostriatal fibers divided into the bundle of Muratoff dorsally above the caudate nucleus and the external capsule. The fibers of the Muratoff bundle ran along the head and body of the caudate nucleus and terminated within its medial part in discrete patches (Fig. 12, sections 2 and 3). The corticostriatal fibers running in the external capsule terminated in the ventromedial part of the putamen, as well as in the nucleus accumbens. Some of the labeled external capsule fibers also terminated in the ventral part of the claustrum as they coursed 


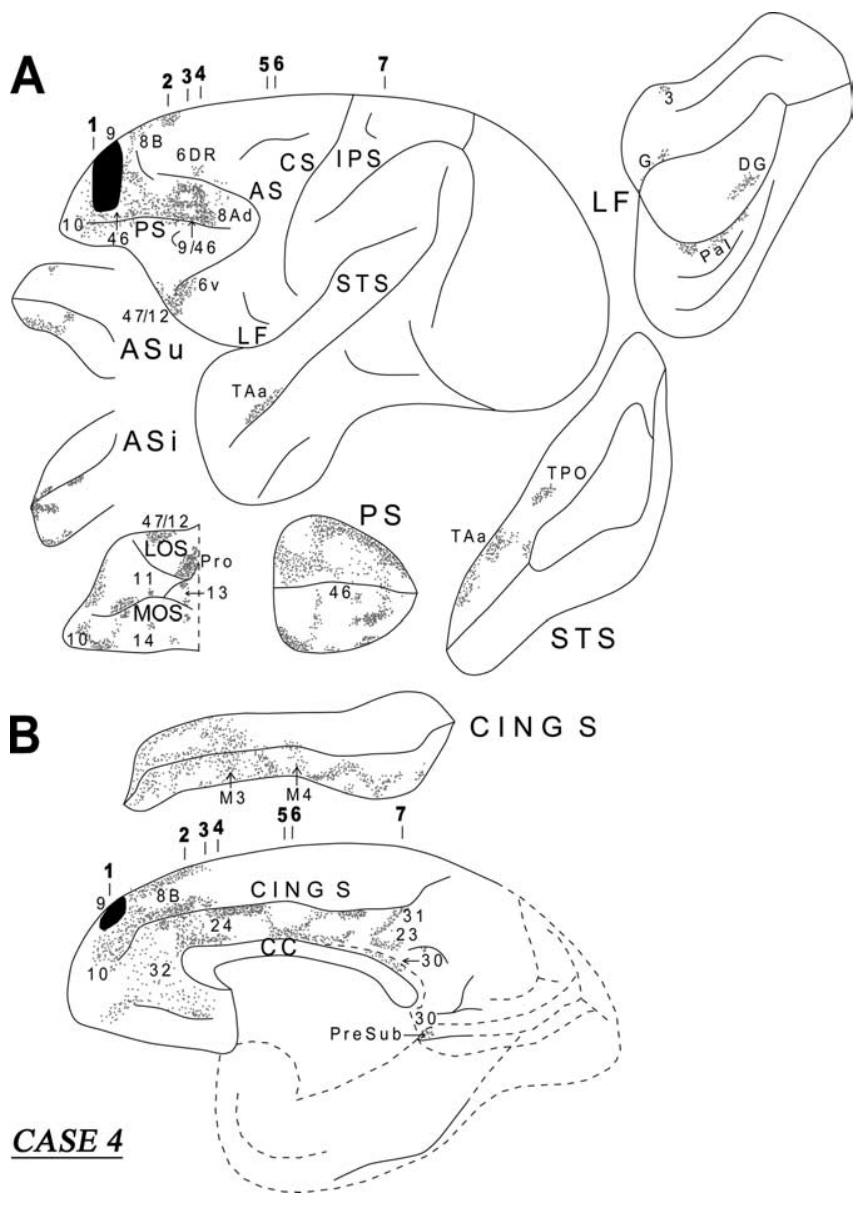

Figure 8. Diagrammatic representation of the unfolded lateral $(\boldsymbol{A})$ and medial $(\boldsymbol{B})$ surfaces of the cerebral hemisphere in case 4 with isotope injection in area 9 (shown in solid black) and the resulting distribution of terminal label (shown as dots). In $A$, the principal sulcus, the upper and inferior limbs of the arcuate sulcus, the lateral fissure, and the superior temporal sulcus have been opened up to show the terminal label in their banks. In $\boldsymbol{B}$, the cingulate sulcus has been opened up. For abbreviations, see Figure 3.

caudally. Another component of labeled fibers from the cord entered the internal capsule. These fibers ran caudally and, at the genu of the internal capsule, this subcortical fiber system divided into two components. The dorsal component penetrated the thalamus and terminated mainly in the dorsomedial nucleus. The other component of these fibers ran ventrally to enter the cerebral peduncle and terminated in the pontine nuclei.

The labeled long association fibers coursed in two directions. The dorsally directed fibers coursed in the cingulate fasciculus and terminated mainly in area 24 in the rostral part of the cingulate gyrus with some fibers extending into the rostral part of area 23 (Fig. 12, sections 2-5). The ventrally directed long association fibers coursed via the extreme capsule and the uncinate bundle (Fig. 12, sections 1-5). The fibers running in the uncinate bundle terminated in the temporopolar proisocortex and area TS1 in the rostral superior temporal gyrus, as well as in the amygdala, predominantly in the intermediate and ventral parts of the basolateral nucleus and, to a lesser extent, the ventral part of the basomedial nucleus. The fibers coursing through the extreme capsule terminated in areas TS2 and TAa of the superior temporal gyrus and area TPO of the superior temporal sulcus.

\section{Case 7 (area 11)}

In this case, the injection involved area 11 in the orbital frontal cortex (Fig. 13A). Adjacent to the injection site, terminal label was observed in ventral area 46,47/12, and ventral area 10 (Fig. $13 A$ ). Caudally directed fibers coursed in the white matter of the frontal lobe and terminated in areas 13 and 14 of the orbital frontal cortex and in ventrolateral frontal cortex in areas $47 / 12$, $45,9 / 46 \mathrm{v}$, and ProM. Other fibers terminated in the rostral insula and the orbital proisocortex. Within the dorsal frontal lobe, one group of fibers coursed dorsally and entered the cingulate fasciculus. These fibers coursed caudally to terminate in the ventral part of area 24.

Distinct clusters of fibers emerged from the injection site as a cord and occupied the central part of the white matter of the frontal lobe. The most medial fibers entered the ventral portion of the genu of the corpus callosum to terminate in the opposite hemisphere (Fig. 14, section 3). Another cluster of fibers entered the anterior limb of the internal capsule and coursed caudally to terminate in the dorsomedial thalamic nucleus. At the level of the anterior horn of the lateral ventricle, yet another cluster of labeled fibers surrounded the head of the caudate nucleus entering the Muratoff bundle and the external capsule (Fig. 14, sections 3, 4). These two striatal fiber systems gave rise to terminations in the head of the caudate nucleus and the ventral part of the putamen, respectively. Some of the labeled fibers from the external capsule and the extreme capsule terminated in the ventral part of the claustrum.

The labeled long association fibers coursed caudally via the uncinate fasciculus and the extreme capsule (Fig. 14, sections 5, 6). The uncinate fasciculus fibers coursed around the limen insulae and terminated in the perirhinal cortex (area 35) and the basolateral nucleus of the amygdala, as well as in areas TL, Pro and rostral TEa and TE1 of the inferotemporal cortex (Figs. 13B, 14, section 6). Fibers from the extreme capsule terminated in the frontal operculum involving the gustatory cortex (Fig. 14, section 5). Further caudally, extreme capsule fibers entered the white matter of the superior temporal sulcus and terminated in area IPa (Fig. 13A). The diagram in the inset of Figure 14 summarizes schematically the two long association pathways originating from area 11 , namely the uncinate fasciculus and the extreme capsule fiber system.

\section{Overall organization of cortical fibers}

There are some general principles of organization of the long association fibers as they enter the white matter immediately below a particular prefrontal cortical area and as they get organized into the fiber bundles demonstrated here. These principles apply to all prefrontal cortical areas examined in this study and in a previous study that examined the fiber bundles originating from the caudal prefrontal region (Petrides and Pandya, 2006). The local association fibers (i.e., fibers to other frontal areas) occupy the space just below the gray matter of the cortical area of origin. In coronal sections, underneath these locally directed fibers, the long association fibers appear diffusely organized, whereas the commissural and subcortical fibers appear as a compact cord-like bundle. The compact cord-like bundle separates into a commissural part that is directed medially toward the genu and the rostrum of the corpus callosum and a subcortical bundle that then splits into various segments. One segment of fibers enters the subcallosal fascicle (Muratoff bundle) and the external capsule and terminates in the striatum (Yeterian and Pandya, 1991). Another segment enters the internal capsule and terminates in the diencephalon (Siwek and Pandya, 1991) and the basis pontis (Schmahmann and Pandya, 1997). The long association fibers, as they course away from the region of origin, are gradually organized into the cingulate fasciculus, the uncinate fasciculus, and the extreme capsule fiber system that are discussed below. 


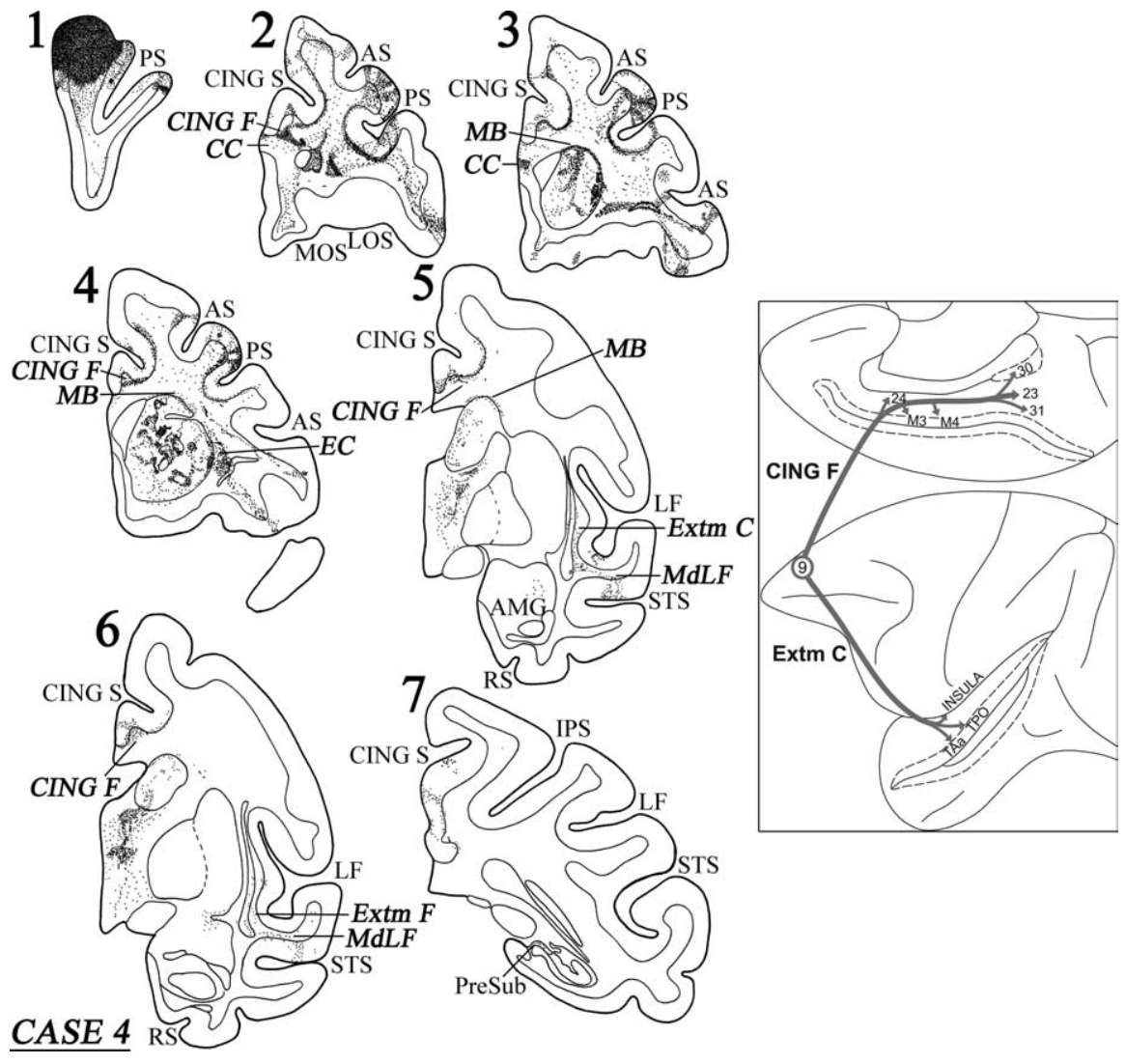

Figure 9. Coronal sections (1-7), at the levels indicated in Figure $8 A$, show the injection site and the labeled pathways in the white matter in case 4. The inset summarizes schematically the origin, trajectories, and terminations of the long association pathways observed in this case. For abbreviations, see Figure 3.

Because there is the issue of whether there are fibers from the orbital and medial prefrontal cortex that enter the fornix to reach the limbic region of the medial temporal lobe (Cavada et al., 2000; Saunders and Aggleton, 2007), we examined our material for labeled fibers in the fornix. In our material, in which the injections were placed in the rostral part of the orbital and medial prefrontal cortex, no labeled fibers were noted in the fornix, suggesting that, if such connections exist, they must originate in the caudal region of the orbital and medial frontal cortex.

\section{Cingulate fasciculus}

The present observations show that efferent association fibers from the medial and lateral parts of the rostral prefrontal cortex course via the cingulate fasciculus and terminate in various parts of the cingulate and retrosplenial cortex. According to Dejerine (1895), the term cingulum of Burdach refers to a long association fascicle coursing within the white matter of the cingulate gyrus and the parahippocampal gyrus. After reviewing previous work and his own, Dejerine (1895) concluded that, in coronal sections stained for fibers, the cingulum is clearly identified as a darkly stained pear-shaped fascicle limited to the ventral-most part of the cingulate white matter just above the corpus callosum. Mufson and Pandya (1984), on the basis of experimental material in the macaque monkey, concluded that the cingulum, as defined above, consists mainly of subcortical afferent and efferent fibers (thalamic, striatal, pontine). The long association fibers that enter the white matter of the cingulate gyrus occupy a more dorsal and medial position around the subcortical fibers and originate from parietal, frontal, temporal, and cingulate cortical regions. We restricted the term cingulate fasciculus to these association fibers. It should be noted that the cingulate fasciculus is a bidirectional association pathway, because the cingulate cortex and the retrosplenial cortex project back to the same prefrontal cortical regions that send efferents to them (Morris et al., 1999a; Petrides and Pandya, 1999).

\section{Uncinate fasciculus}

This fasciculus was first described by Reil and was referred to by Burdach as the "fasciculus unciformis" (Dejerine, 1895). The uncinate fibers extend from the anterior temporal lobe to the orbital frontal cortex, forming a sharp arch at the level of the most ventral and anterior part of the insula (limen insulae). Experimental anatomical studies in the macaque monkey have shown that fibers running through the uncinate fasciculus connect the orbitofrontal cortex with the temporopolar region, the rostral parahippocampal gyrus (entorhinal/perirhinal region), and the amygdala (Van Hoesen et al., 1975; Petrides and Pandya, 1988; Carmichael and Price, 1995). The present data show that fibers from areas 10, 32, and 11 course through the uncinate fasciculus to terminate in the rostral temporal cortex and the amygdala. Klingler and Gloor (1960), based on dissection methods in the human brain, reported that the orbital frontal cortex is connected with the amygdala and other parts of the anterior temporal region via the uncinate fasciculus. These findings may appear to be in conflict with the observation by Ungerleider et al. (1989) that transection of the uncinate fasciculus leaves intact connections between the amygdala and the prefrontal cortex and the finding by Eacott and Gaffan (1992) that reward information (which presumably originates in the amygdala) can reach the frontal cortex after such transections. This apparent discrepancy is probably a result of differences in the definition of the uncinate fasciculus. The fibers connecting the amygdala with the orbital and medial frontal cortex course at the level of the limen insulae where they exhibit a marked curvature (hence the name uncinate) and remain below the extreme capsule. The fibers transected in the studies by Ungerleider et al. (1989) and Eacott and Gaffan (1992), and which left the amygdalo-frontal connections intact, were located above the limen insulae in the ventral part of the extreme capsule. Because these fibers are running in the extreme capsule, we classify them as part of the extreme capsule fiber system and not the uncinate fasciculus. Such transections would spare the amygdalo-prefrontal connections, which run more ventrally in the limen insulae. We define the uncinate fasciculus as only those hook-like (i.e., uncinate) temporofrontal fibers that run at the level of the limen insulae and below the extreme capsule. Note that in the white matter of the orbital frontal lobe, fibers that run in the limen insulae (i.e., the uncinate fibers) are located medial to fibers that run in the extreme capsule above the limen insulae (i.e., the extreme capsule fibers) (Fig. 14, sections 3-5).

\section{Extreme capsule fiber system}

There is considerable confusion with regard to the fibers that enter the extreme capsule. According to Dejerine (1895), the extreme capsule comprises mainly short association fibers linking 


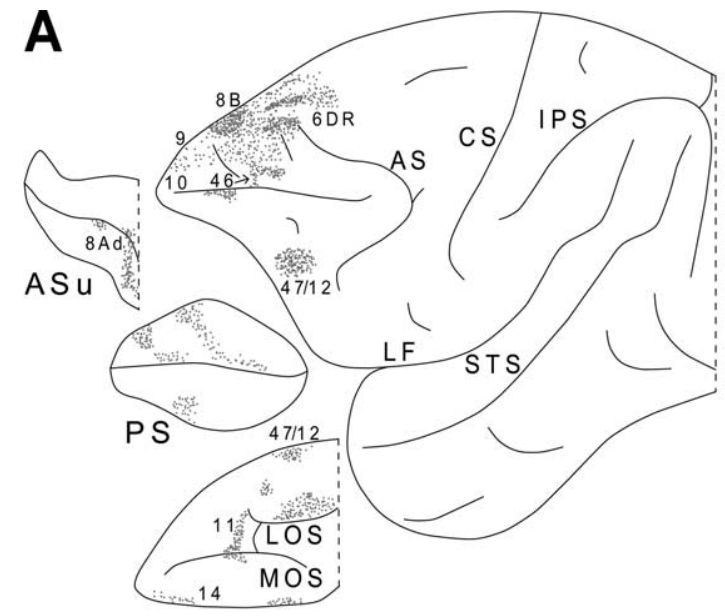

B

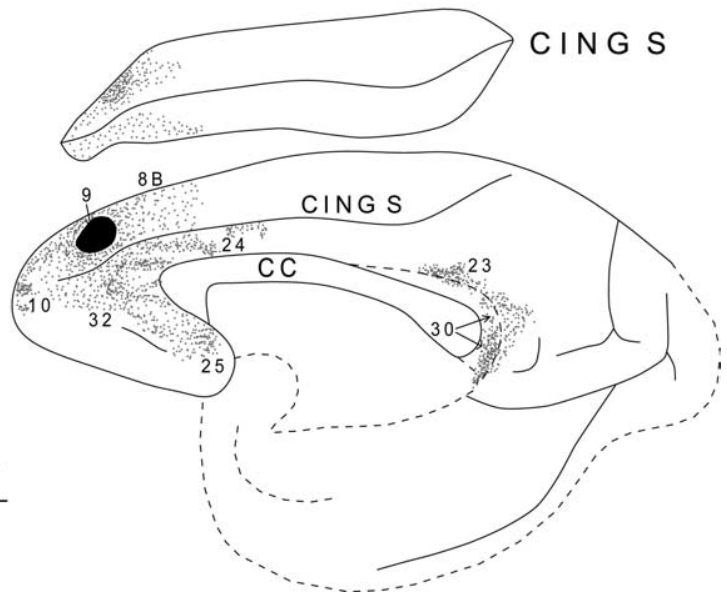

Figure 10. Diagrammatic representation of the unfolded lateral $(\boldsymbol{A})$ and medial $(\boldsymbol{B})$ surfaces of the cerebral hemisphere in case 5 with isotope injection in the medial part of area 9 (shown in solid black) and the resulting distribution of terminal label (shown as dots). In $\boldsymbol{A}$, the principal sulcus and the upper limb of the arcuate sulcus and, in $\boldsymbol{B}$, the cingulate sulcus have been opened up to show the terminal label in their banks. For abbreviations, see Figure 3.

the gyri of the insula. In a survey of white matter pathways, Crosby (1982) stated that the extreme capsule comprises association fibers linking frontal cortex with temporal cortex, parietal cortex with temporal cortex, as well as these cortical regions with the insula. The present data indicate that fibers originating from the rostral prefrontal cortex and that are directed to the insula and the temporal lobe course through the extreme capsule. Those prefrontal extreme capsule fibers that enter the white matter of the superior temporal gyrus continue, as part of the MdLF, to terminate in the midportion of the superior temporal gyrus and the superior temporal sulcus. Whereas the prefrontal fibers that enter the uncinate fasciculus project to the temporal pole and amygdala, the fibers running in the extreme capsule are directed to the rostral part of the insula, the superior temporal gyrus, and the multimodal areas of the superior temporal sulcus.

\section{Discussion}

The present study demonstrates that the long efferent association fibers, which convey rostral prefrontal control over posterior cortical areas, are organized into three distinct streams: (1) a dorsomedial limbic stream via the cingulate fasciculus that targets the anterior and posterior cingulate cortex and the retrosplenial cortex; (2) a ventral limbic stream via the uncinate fasciculus that

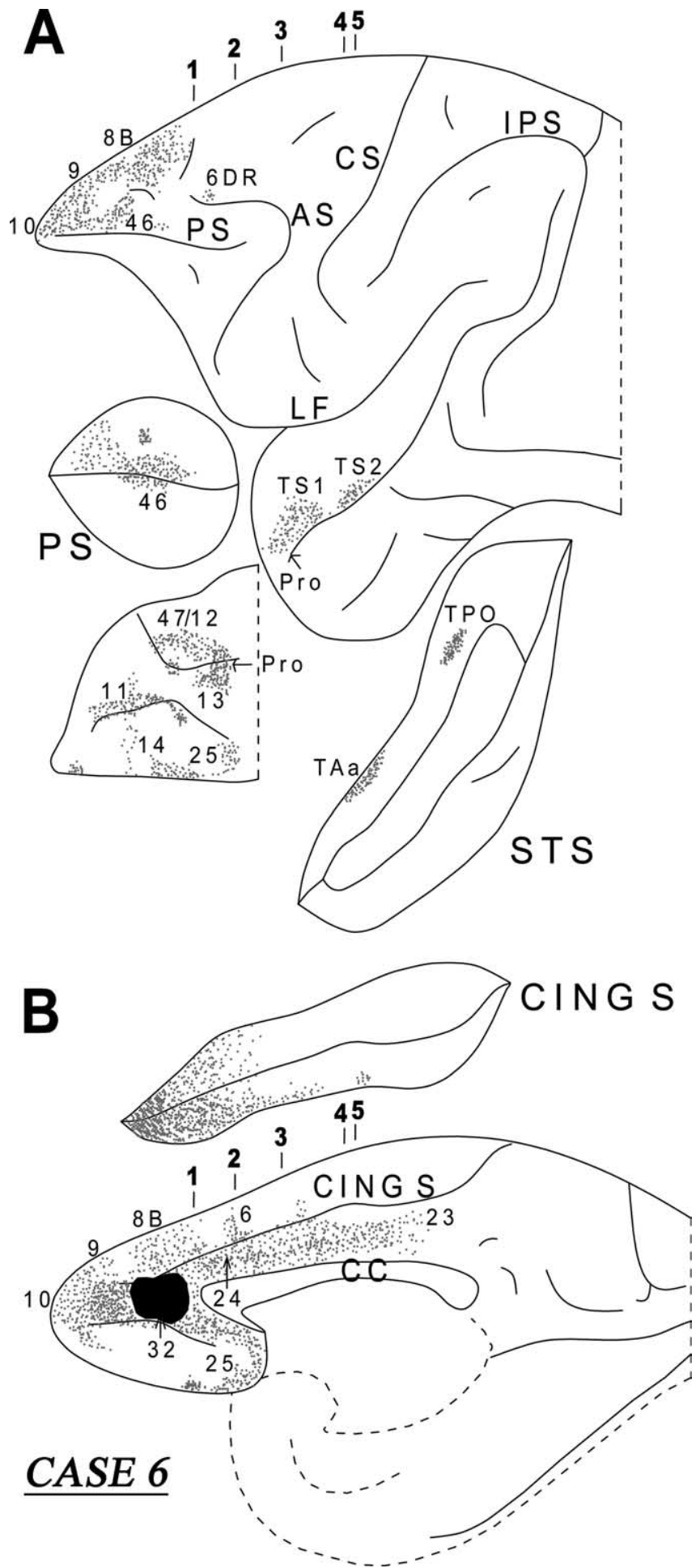

Figure 11. Diagrammatic representation of the unfolded lateral $(\boldsymbol{A})$ and medial $(\boldsymbol{B})$ surfaces of the cerebral hemisphere in case 6 with isotope injection in area 32 (shown in solid black) and the resulting distribution of terminal label (shown as dots). In $\boldsymbol{A}$, the principal sulcus and the superior temporal sulcus and, in $\boldsymbol{B}$, the cingulate sulcus have been opened up to show the terminal label in their banks. For abbreviations, see Figure 3.

targets the temporopolar proisocortex, the rostral-most superior temporal gyrus, and the amygdala; and (3) a lateral stream of fibers that enters the extreme capsule and targets the midportion of the superior temporal gyrus and adjacent multisensory areas within the upper bank of the superior temporal sulcus (Figs. 15, 


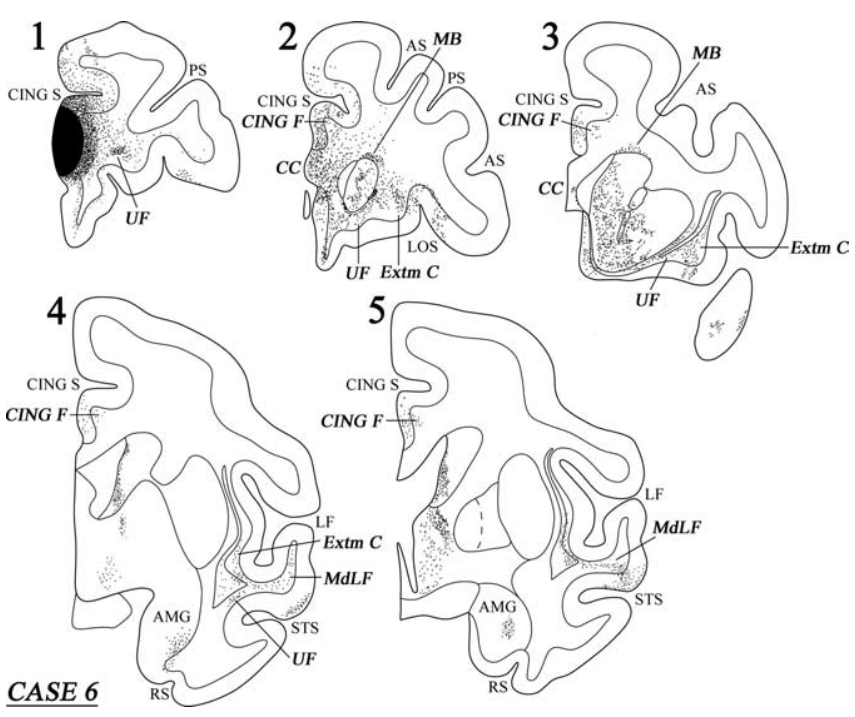

Figure 12. Coronal sections (1-5), at the levels indicated in Figure $11 A$, show the injection site and the labeled pathways in the white matter in case 6 . For abbreviations, see Figure 3.

16). Note that these same fasciculi contain afferent association fibers that provide input to these prefrontal areas from postRolandic cortical regions (for review, see Petrides and Pandya, 2002).

From the most rostral part of the prefrontal cortex, namely area 10, a group of fibers courses dorsally and enters the cingulate fasciculus. These fibers terminate in area 24 of the rostral cingulate cortex, in areas 23 and 31 of the posterior cingulate cortex, and in the retrosplenial area 30 (Figs. 4, 16). Another group of fibers from area 10 courses, ventrocaudally, to form the uncinate fasciculus, which terminates in the temporopolar proisocortex, the amygdala, and in area TS1 in the rostral part of the superior temporal gyrus (Figs. 4, 15). Finally, a third group of fibers enters the extreme capsule and courses caudally in the white matter of the superior temporal gyrus, merging with the fibers of the MdLF, to terminate in areas TS2 and TS3 of the superior temporal gyrus and in areas TAa and TPO of the superior temporal sulcus (Figs. $4,16)$. Some of the extreme capsule fibers terminate in the ventral part of the insula and in areas Pro and $\mathrm{PaI}$ in the circular sulcus (Fig. 3A).

The long association fibers originating within area 9 were similar to those of area 10 but with some notable differences. Efferent corticocortical fibers from area 9 use the cingulate fasciculus and the extreme capsule, but not the uncinate fasciculus, to convey control over posterior cortical areas. As was the case with area 10, fibers originating in medial and lateral area 9 enter the cingulate fasciculus and terminate in the anterior (area 24) and posterior (areas 23 and 31) cingulate cortex, as well as in the retrosplenial area 30 (Figs. 6-10). However, area 9 fibers coursing in the cingulate fasciculus also terminate in the motor cingulate areas (M3 and M4) (Figs. 6-9). The fibers from area 9 that are running though the extreme capsule terminate in area $\mathrm{PaI}$ in the circular sulcus, the dysgranular insula, and in superior temporal areas TAa and TPO (Figs. 6-9). When the injection was restricted to the part of area 9 that lies on the medial surface of the frontal lobe (Fig. 10, case 5), no connections were observed to be running through the extreme capsule to the superior temporal region, suggesting that the latter projection originates only from the lateral part of area 9 .

The specificity of the connections of area 10 and area 9 with the rostral part of the superior temporal gyrus and the adjacent
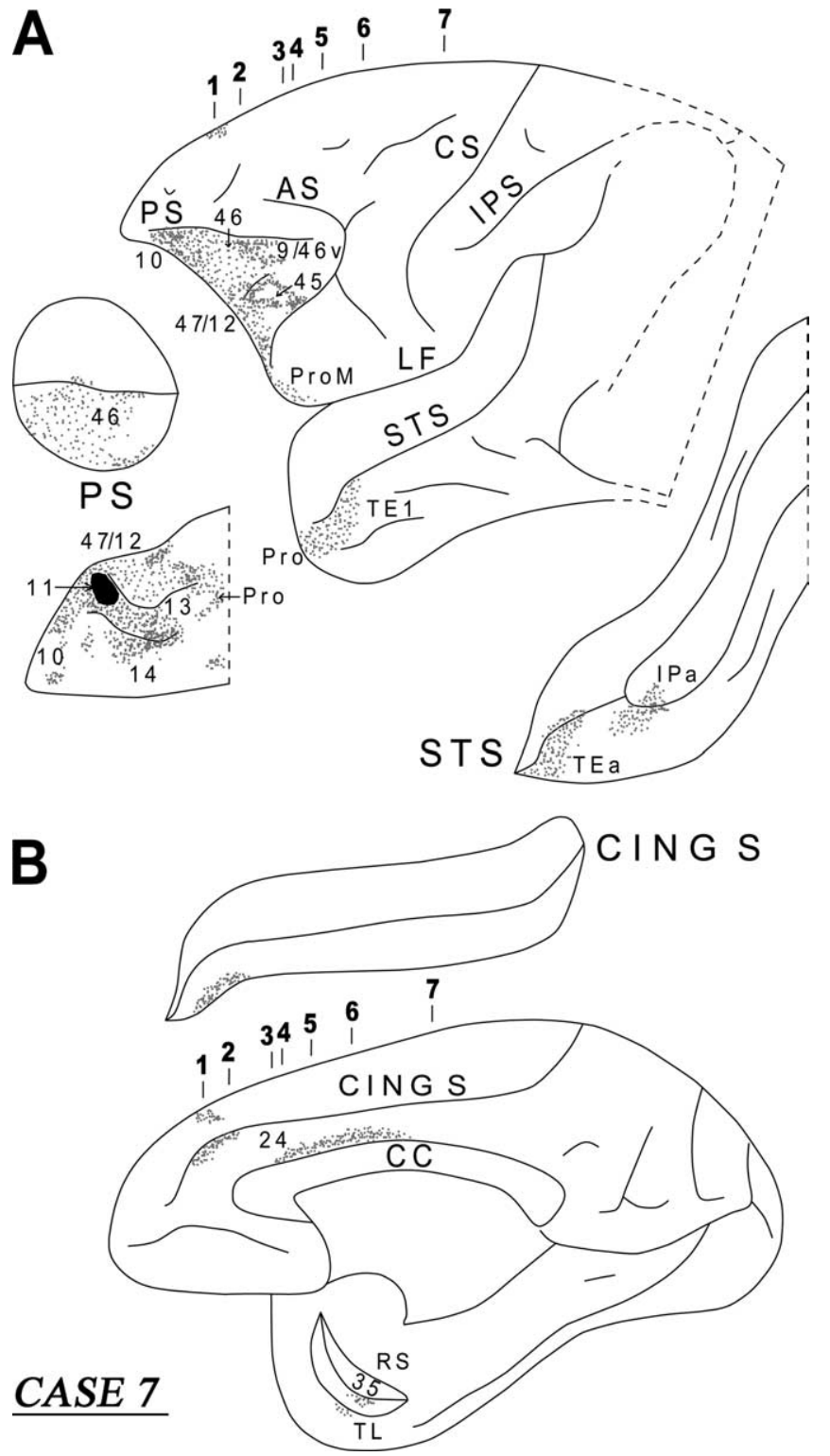

Figure 13. Diagrammatic representation of the unfolded lateral $(\boldsymbol{A})$ and medial $(\boldsymbol{B})$ surfaces of the cerebral hemisphere in case 7 with isotope injection in area 11 (shown in solid black) and the resulting distribution of terminal label (shown as dots). In $A$, the principal sulcus and the superior temporal sulcus and, in $\boldsymbol{B}$, the cingulate sulcus have been opened up to show the terminal label in their banks. For abbreviations, see Figure 3.

superior temporal sulcus is highlighted when the connections of orbitofrontal area 11 (which lies just caudal to area 10 on the orbital surface) are examined (Figs. 13, 14). Long association fibers from area 11 course through the uncinate fasciculus and the extreme capsule. The fibers running through the uncinate fasciculus terminate in the perirhinal cortex (area 35), area TL, the amygdala, the temporopolar cortex, and adjacent rostral inferotemporal area TE1, whereas those fibers running through the extreme capsule terminate in area IPa in the depth of the superior temporal sulcus. Thus, unlike the connections of areas 10 and 9 that target auditory and multisensory areas in the superior temporal region, area 11 connections target primarily visual areas in the rostral inferotemporal cortex. The fibers coursing through the extreme capsule terminate in the gustatory cortex in the lateral fissure, as well as in area IPa and TEa in the depth and lower bank of the superior temporal sulcus (Fig. 14, sections 6, 7). The 

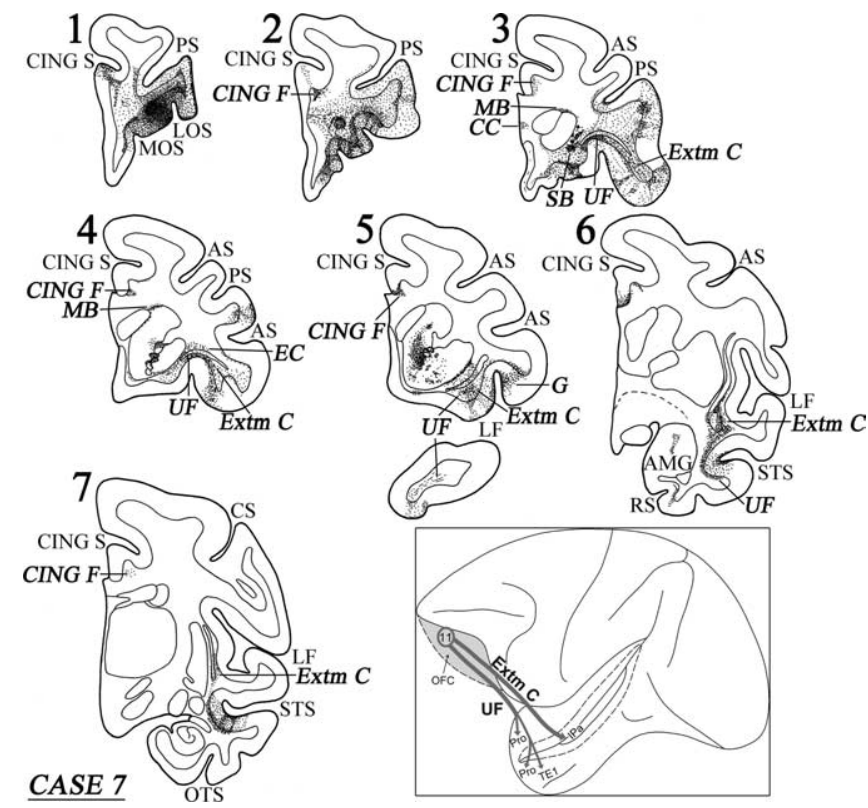

Figure 14. Coronal sections (1-7), at the levels indicated in Figure 13A, show the injection site and the labeled pathways in the white matter in case 7 . The inset summarizes schematically the origin, trajectories, and terminations of the long association pathways observed in this case. For abbreviations, see Figure 3.

few fibers that enter the cingulate fasciculus are directed to the rostral cingulate gyrus.

\section{Functional considerations}

It is important to note that neither area 10 nor area 9 project to any part of the parietal cortex, the visual areas of the occipital cortex and the inferotemporal cortex (Figs. 3-10). Thus, the rostral prefrontal cortex does not interact directly with the occipitoparietal cortical areas that process visuospatial information or the occipitotemporal cortical areas that process visual object information (Ungerleider and Mishkin, 1982) or the parietal areas involved in action in space (Lacquaniti et al., 1995; Gallese et al., 2002). This fact stands in striking contrast with the welldocumented massive and bidirectional connections that link the caudal lateral prefrontal (areas $8 \mathrm{~A}$ and $8 \mathrm{~B}$ ) and the midlateral prefrontal (areas 9/46 and 45) cortex with parts of the occipital, parietal, and inferotemporal cortex (Cavada and GoldmanRakic, 1989; Andersen et al., 1990; Petrides and Pandya, 1984, 1999, 2006). These caudal prefrontal to parietal connections underlie attentional modulations of visuospatial, auditory spatial, and action-in-space information processing (i.e., processing subserved by specific parts of the parietal and adjacent parietotemporal cortex) (Barcelo et al., 2000; Nobre et al., 2004). Thus, the rostral prefrontal cortex, by not interacting with these posterior cortical areas, does not regulate attention to events occurring in external space. This is the domain of the caudal prefrontal cortical region via special pathways such as the three branches of the superior longitudinal fasciculus and the fronto-occipital fasciculus (Petrides and Pandya, 2006).

The present results clearly indicate that the rostral prefrontal cortex (both areas 10 and 9) is preferentially connected with the midsection of the auditory superior temporal cortex and the multisensory processing region of the superior temporal sulcus (areas TAa and TPO). It is known that these auditory and multisensory processing areas project back to the rostral prefrontal cortex (Petrides and Pandya, 1988; Romanski et al., 1999a,b). Although the precise role of these multisensory processing areas remains to be elucidated, it is clear that they play a major role in the highest level of integration of information coming from visual, auditory, and somatic sensory systems to achieve amodal, abstract, conceptual interpretation of the environment. For instance, there is evidence from single neuron recording in monkeys (Perrett et al., 1985) and imaging in human subjects (Wicker et al., 2003) that the multisensory region in the superior temporal sulcus is involved in the processing of the intentions of other individuals as this is expressed in gaze behavior. The significant connections here demonstrated between area 9 and the superior temporal sulcus may be necessary for the suggested role of area 9 in interpreting the intentions of others (Frith and Frith, 2003). Thus, the rostral prefrontal cortex is exerting its control, via the extreme capsule, on the most highly integrated cortical information, and these connections may be the anatomical basis for the suggested role of the rostral prefrontal cortex in influencing abstract information processing (Christoff et al., 2003) and the integration of the outcomes of multiple cognitive operations (Ramnani and Owen, 2004; Petrides, 2005).

Additionally, the connections to the multisensory temporal region referred to above, the rostral prefrontal region exerts influence on the caudal orbitofrontal cortex via local connections, on the amygdala via the uncinate fasciculus, and on the cingulate cortex via the cingulate fasciculus. All these target structures have been linked with the processing of emotional information (Rolls, 1999) and, therefore, these efferent connections of the rostral prefrontal cortex provide the anatomical basis for prefrontal control over the emotional and motivational state of the individual. Several recent functional neuroimaging studies have emphasized the role of the rostral prefrontal region in the ability to reflect on one's own and the other's emotional states (Damasio et al., 2000; Kelly et al., 2003).

Understanding the function of the most rostral part of the prefrontal cortex remains elusive despite the recent progress that has been made. The present study demonstrates the specific pathways that underlie cognitive control operations emanating from the rostral prefrontal region. The cingulate and uncinate fasciculi enable interactions between the rostral prefrontal cortex with limbic structures, such as the cingulate cortex, the temporopolar proisocortex, and the amygdala and therefore provide the basis for control over one's own and other persons' motivational/emotional states. In contrast, the extreme capsule fiber system permits interactions between the lateral rostral prefrontal region and auditory and multisensory cognitive processing in the temporal lobe, namely cognitive processing underlying conceptual knowledge and abstract thought. There appears to be a caudal-torostral gradient in lateral prefrontal functional organization: the caudal prefrontal region via its own unique efferent pathways (i.e., the superior longitudinal and fronto-occipital fasciculi) modulates attention to the external environment and action within it, but, as one proceeds rostrally, control is shifted away from the external environment and is focused on abstract multisensory processing and internal emotional states (Petrides, 2005).

\section{References}

Andersen RA, Asanuma C, Essick G, Siegel RM (1990) Corticocortical connections of anatomically and physiologically defined subdivisions within the inferior parietal lobule. J Comp Neurol 296:65-113.

Barbas H (1988) Anatomic organization of basoventral and mediodorsal visual recipient prefrontal regions in the rhesus monkey. J Comp Neurol 276:313-342.

Barbas H, Mesulam M-M (1981) Organization of afferent input to subdivisions of area 8 in the rhesus monkey. J Comp Neurol 200:407-431. 

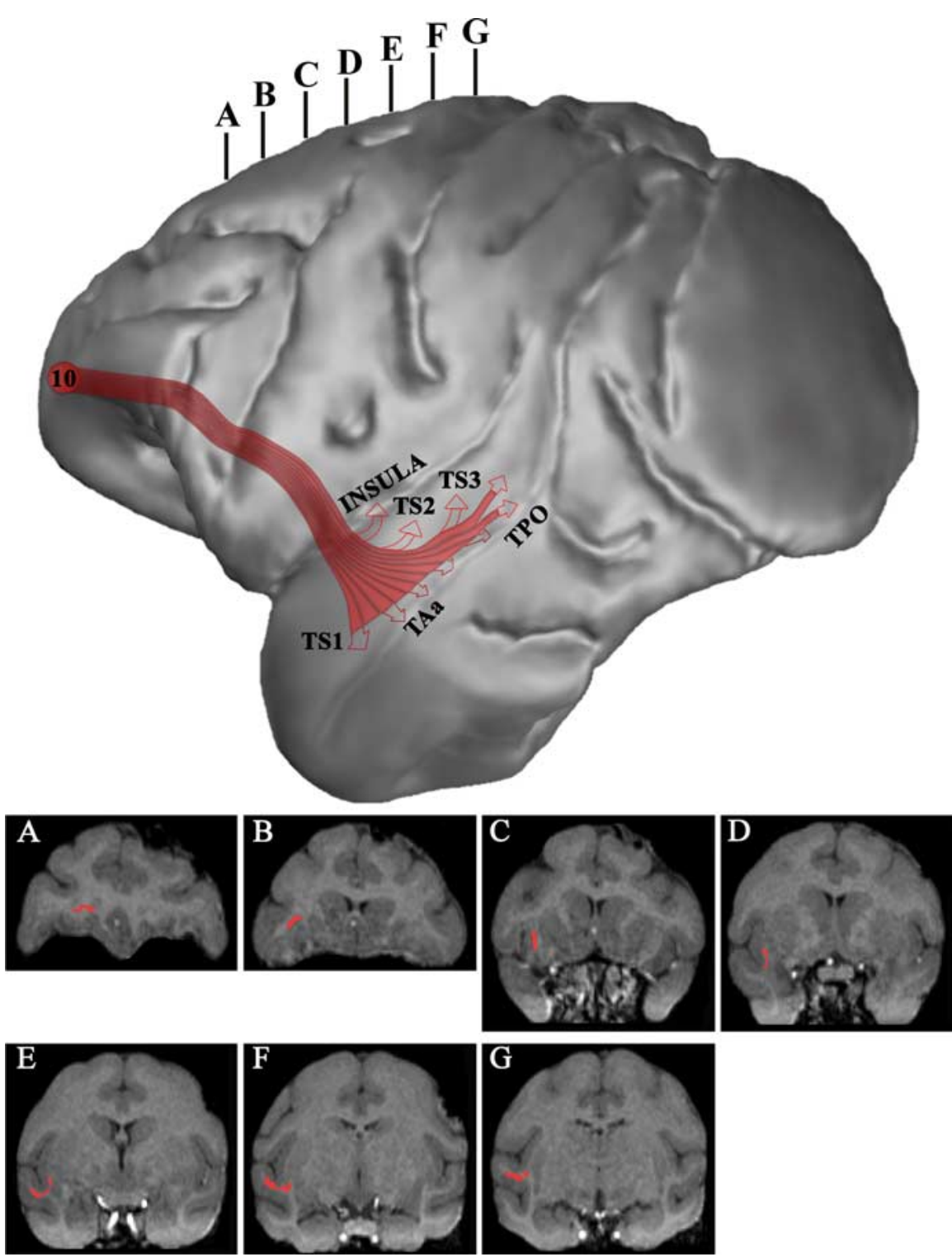

Figure 15. Three-dimensional reconstruction of the MRI of a macaque monkey brain to illustrate the course of the extreme capsule efferent fiber system (in red) that connects frontopolar area 10 with the midportion of the superior temporal gyrus and sulcus and the insula. The accompanying coronal sections $(\boldsymbol{A}-\boldsymbol{G})$ were taken at the levels indicated at the top of the lateral hemisphere to show the course of these pathways as seen in coronal planes.

Barcelo F, Suwazono S, Knight RT (2000) Prefrontal modulation of visual processing in humans. Nat Neurosci 3:399-403.

Carmichael ST, Price JL (1995) Limbic connections of the orbital and medial prefrontal cortex in macaque monkeys. J Comp Neurol 363:615-641.

Carmichael ST, Price JL (1996) Connectional networks within the orbital and medial prefrontal cortex of macaque monkeys. J Comp Neurol 371:179-207.

Cavada C, Goldman-Rakic PS (1989) Posterior parietal cortex in the rhesus monkey: II. Evidence for segregated corticocortical networks linking sensory and limbic areas with the frontal lobe. J Comp Neurol 287:422-445.

Cavada C, Compañy T, Tejedor J, Cruz-Rizzolo RJ, Reinoso-Suárez F (2000) The anatomical connections of the macaque monkey orbitofrontal cortex. A review. Cereb Cortex 10:220-242.

Christoff K, Ream JM, Geddes LP, Gabrieli JD (2003) Evaluating selfgenerated information: anterior prefrontal contribution to human cognition. Behav Neurosci 117:1161-1168.

Cowan WM, Gottlieb DI, Hendrickson AE, Price JL, Woolsey TA (1972) The autoradiographic demonstration of axonal connections in the central nervous system. Brain Res 37:21-51.

Crosby EC (1982) Section C. White matter of hemispheres. In: Comparative

put 2359:160-169. correlative neuroanatomy of the vertebrate telencephalon (Crosby EC, Schnitzlein HN, eds). New York: Macmillan.

Damasio AR, Grabowski TJ, Bechara A, Damasio H, Ponto LL, Parvizi J, Hichwa RD (2000) Subcortical and cortical brain activity during the feeling of self-generated emotions. Nat Neurosci 3:1049-1056.

Dejerine J (1895) Anatomie des centres nerveux, Vol I. Paris: Rueff.

Eacott MJ, Gaffan D (1992) Inferotemporalfrontal disconnection: the uncinate fascicle and visual associative learning in monkeys. Eur J Neurosci 4:1320-1332.

Frith U, Frith CD (2003) Development and neurophysiology of mentalizing. Philos Trans R Soc Lond B Biol Sci 358:459-473.

Gallese V, Fogassi L, Fadiga L, Rizzolatti G (2002) Action representation and the inferior parietal lobule. In: Attention and performance XIX: common mechanisms in perception and action (Prinz W, Hommel B, eds), pp 335-355. Oxford: Oxford UP.

Gilbert SJ, Spengler S, Simons JS, Steele JD, Lawrie SM, Frith CD, Burgess PW (2006) Functional specialization within rostral prefrontal cortex (area 10): a meta-analysis. J Cogn Neurosci 18:932-948.

Goldman-Rakic PS, Selemon LD, Schwartz ML (1984) Dual pathways connecting the dorsolateral prefrontal cortex with the hippocampal formation and parahippocampal cortex in the rhesus monkey. Neuroscience 12:719-743.

Kelly WM, Macrae CN, Wyland CL, Caglar S, Inati S, Heatherton TP (2003) Finding the self? An event-related fMRI study. J Cogn Neurosci 14:785-794.

Klingler J, Gloor P (1960) The connections of the amygdala and the anterior temporal cortex in the human brain. J Comp Neurol 115:333-369.

Lacquaniti F, Guigon E, Bianchi L, Ferraina S, Caminiti R (1995) Representing spatial information for limb movement: role of area 5 in the monkey. Cereb Cortex 5:391-409.

MacDonald D (1996) MNI-display: Program for display and segmentation of surfaces and volumes. Retrieved September 20, 2007, from http://www.bic.mni.mcgill. ca/software/Display/Display.html.

MacDonald D, Avis D, Evans AC (1994) Multiple surface identification and matching in magnetic resonance images. Vis Biomed Com-

Morris R, Petrides M, Pandya DN (1999a) Architecture and connections of retrosplenial area 30 in the rhesus monkey (macaca mulatta). Eur J Neurosci 11:2506-2518.

Morris R, Pandya DN, Petrides M (1999b) Fiber system linking the middorsolateral frontal cortex with the retrosplenial/presubicular region in the rhesus monkey. J Comp Neurol 407:183-192.

Mufson EJ, Pandya DN (1984) Some observations on the course and composition of the cingulum bundle in the rhesus monkey. J Comp Neurol 225:31-43.

Nauta WJH (1964) Some efferent connections of the prefrontal cortex in the monkey. In: The frontal granular cortex and behavior, Chap 19 (Warren JM, Akert K, eds), pp 397-409. New York: McGraw-Hill.

Nobre AC, Coull JT, Maquet P, Frith CD, Vandenberghe R, Mesulam M-M (2004) Orienting attention to locations in perceptual versus mental representations. J Cogn Neurosci 16:363-373.

Öngür D, An X, Price JL (1998) Prefrontal cortical projections to the hypothalamus in macaque monkeys. J Comp Neurol 401:480-505.

Pandya DN, Sanides F (1973) Architectonic parcellation of the temporal 
operculum in the rhesus monkey and its projection pattern. Z Anat Entwickl Gesch 139:127-161.

Pandya DN, Seltzer B (1982) Intrinsic connections and architectonics of posterior parietal cortex in the rhesus monkey. J Comp Neurol 204:196-210.

Paxinos G, Huang X-F, Toga AW (2000) The rhesus monkey brain in stereotaxic coordinates. San Diego: Academic.

Perrett DI, Smith PAJ, Potter DD, Mistlin AJ, Head AS, Milner AD, Jeeves MA (1985) Visual cells in the temporal cortex sensitive to face view and gaze direction. Proc R Soc Lond B Biol Sci 223:293-317.

Petrides M (2005) Lateral prefrontal cortex: architectonic and functional organization. Philos Trans R Soc Lond B Biol Sci 360:781-795.

Petrides M, Pandya DN (1984) Projections to the frontal cortex from the posterior parietal region in the rhesus monkey. J Comp Neurol 228:105-116.

Petrides M, Pandya DN (1988) Association fiber pathways to the frontal cortex from the superior temporal region in the rhesus monkey. J Comp Neurol 273:52-66.

Petrides M, Pandya DN (1994) Comparative architectonic analysis of the human and the macaque frontal cortex. In: Handbook of neuropsychology, Vol IX (Boller F, Grafman J, eds), pp 17-58. Amsterdam: Elsevier.

Petrides M, Pandya DN (1999) Dorsolateral prefrontal cortex: comparative cytoarchitectonic analysis in the human and the macaque brain and corticocortical connection patterns. Eur J Neurosci 11:1011-1036.

Petrides M, Pandya DN (2002) Association pathways of the prefrontal cortex and functional observations. In: Principles of frontal lobe function, Chap 3 (Stuss DT, Knight RT, eds), pp 31-50. New York: Oxford UP.

Petrides M, Pandya DN (2006) Efferent ssociation pathways originating in the caudal prefrontal cortex in the macaque monkey. J Comp Neurol 498:227-251.

Ramnani N, Owen AM (2004) Anterior prefrontal cortex: insights into function from anatomy and neuroimaging. Nat Rev Neurosci 5:184-194.

Rempel-Clower NL, Barbas H (1998) Topographic organization of connections between the hypothalamus and prefrontal cortex in the rhesus monkey. J Comp Neurol 398:393-419.

Rolls ET (1999) The brain and emotion. Oxford: Oxford UP.

Romanski LM, Bates JF, Goldman-Rakic PS (1999a) Auditory belt and parabelt projections to the prefrontal cortex in the rhesus monkey. J Comp Neurol 403:141-157.

Romanski LM, Tian B, Fritz J, Mishkin M, Goldman-Rakic PS, Rauschecker JP (1999b) Dual streams of auditory afferents target multiple domains in the primate prefrontal cortex. Nat Neurosci 2:1131-1136.

Rosene DL, Pandya DN (1983) Architectonics and connections of the posterior parahippocampal gyrus in the rhesus monkey. Soc Neurosci Abstr 9:222.

Saunders RC, Aggleton JP (2007) Origin and topography of fibers contributing to the fornix in macaque monkeys. Hippocampus 17:396-411.

Schmahmann JD, Pandya DN (1997) Anatomic organization of basilar pontine projections from the prefrontal cortices in the rhesus monkey. J Neurosci 17:438-458.

Seltzer B, Pandya DN (1978) Afferent cortical connections and architectonics of the superior temporal sulcus and surrounding cortex in the rhesus monkey. Brain Res 149:1-24.
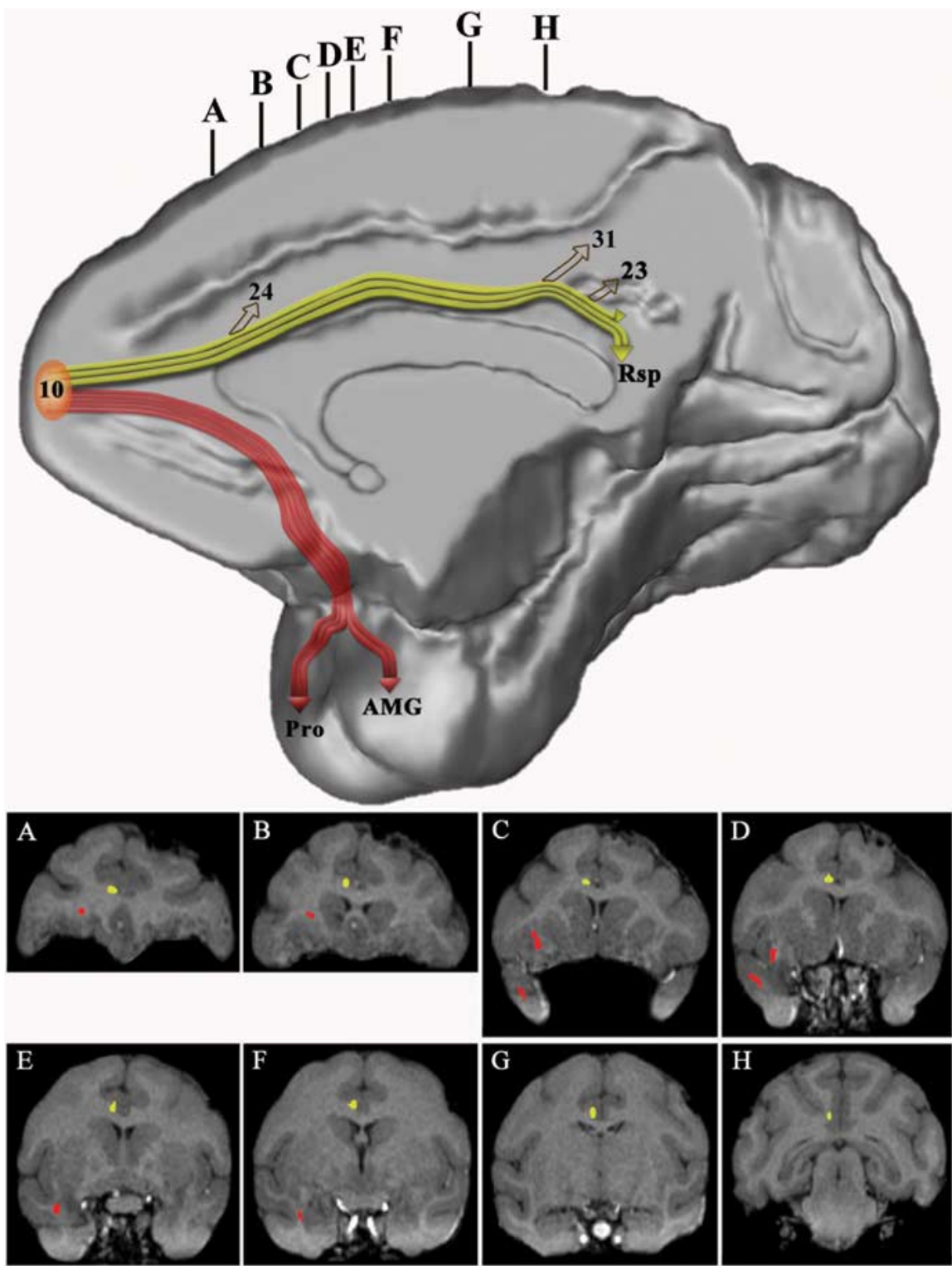

Figure 16. Three-dimensional reconstruction of the MRI of a macaque monkey brain to illustrate the course of the cingulate ficulus (in yellow) that connects frontopolar area 10 with rostral and caudal cingulate cortex and the retrosplenial cortex and The accompanying coronal sections $(\boldsymbol{A}-\boldsymbol{H})$ were taken at the levels indicated at the top of the medial hemisphere to show the course of these pathways as seen in coronal planes.

Siwek DF, Pandya DN (1991) Prefrontal projections to the mediodorsal nucleus of the thalamus in the rhesus monkey. J Comp Neurol 312:509-524.

Ungerleider LG, Mishkin M (1982) Two cortical visual systems. In: Analysis of visual behavior (Ingle DJ, Goodale MA, Mansfield RJW, eds), pp 549586. Cambridge, MA: MIT.

Ungerleider LG, Gaffan D, Pelak VS (1989) Projections from inferior temporal cortex to prefrontal cortex via the uncinate fascicle in rhesus monkeys. Exp Brain Res 76:473-484.

Van Hoesen GW, Pandya DN, Butters N (1975) Some connections of the entorhinal (area 28) and perirhinal (area 35) cortices of the rhesus monkey. II. Frontal lobe afferents. Brain Res 95:25-38.

Vogt BA, Pandya DN, Rosene DL (1987) Cingulate cortex of the rhesus monkey: I. Cytoarchitecture and thalamic afferents. J Comp Neurol 262:256-270.

Wicker B, Perrett DI, Baron-Cohen S, Decety J (2003) Being the target of another's emotion: a PET study. Neuropsychologia 41:139-146.

Yeterian EH, Pandya DN (1991) Prefrontostriatal connections in relation to cortical architectonic organization in rhesus monkey. J Comp Neurol 312:43-67. 